\title{
Incidence of Differentiation Syndrome Associated with Treatment Regimens in Acute Myeloid Leukemia: A Systematic Review of the Literature
}

\author{
Lucia Gasparovic ${ }^{1}$, Stefan Weiler ${ }^{1,2}$, Lukas Higi ${ }^{1}$ and Andrea M. Burden ${ }^{1, *(1)}$ \\ 1 Institute of Pharmaceutical Sciences, Department of Chemistry and Applied Biosciences, ETH Zurich, \\ 8093 Zurich, Switzerland; glucia@student.ethz.ch (L.G.); Stefan.weiler@toxinfo.ch (S.W.); \\ lhigi@student.ethz.ch (L.H.) \\ 2 National Poisons Information Centre, Tox Info Suisse, Associated Institute of the University of Zurich, \\ 8032 Zurich, Switzerland \\ * Correspondence: andrea.burden@pharma.ethz.ch; Tel.: +41-76-685-22-56
}

Received: 30 August 2020; Accepted: 14 October 2020; Published: 18 October 2020

\begin{abstract}
Differentiation syndrome (DS) is a potentially fatal adverse drug reaction caused by the so-called differentiating agents such as all-trans retinoic acid (ATRA) and arsenic trioxide (ATO), used for remission induction in the treatment of the M3 subtype of acute myeloid leukemia (AML), acute promyelocytic leukemia (APL). However, recent DS reports in trials of isocitrate dehydrogenase (IDH)-inhibitor drugs in patients with IDH-mutated AML have raised concerns. Given the limited knowledge of the incidence of DS with differentiating agents, we conducted a systematic literature review of clinical trials with reports of DS to provide a comprehensive overview of the medications associated with DS. In particular, we focused on the incidence of DS reported among the IDH-inhibitors, compared to existing ATRA and ATO therapies. We identified 44 published articles, encompassing 39 clinical trials, including 6949 patients. Overall, the cumulative incidence of DS across all treatment regimens was $17.7 \%$. Incidence of DS was notably lower in trials with IDH-inhibitors $(10.4 \%)$ compared to other regimens, including ATRA and/or ATO (15.4-20.6\%). Compared to other therapies, the median time to onset was four times longer with IDH-inhibitors (48 vs. 11 days). Treating oncologists should be mindful of this potentially fatal adverse drug reaction, as we expect the current trials represent an underestimation of the actual incidence.
\end{abstract}

Keywords: differentiation syndrome; retinoic acid syndrome; differentiating agents; acute myeloid leukemia; non-M3 acute myeloid leukemia; acute promyelocytic leukemia; systematic review

\section{Introduction}

Differentiation syndrome (DS) is a potentially fatal adverse drug reaction caused by the so-called differentiating agents, such as all-trans retinoic acid (ATRA) and arsenic trioxide (ATO) drugs, which control cellular differentiation and proliferation. The primary use of ATRA and ATO is for remission induction in treating the M3 subtype of acute myeloid leukemia (AML), acute promyelocytic leukemia (APL) [1,2]. APL accounts for approximately 5 to $20 \%$ of all AML cases, and age at diagnosis is significantly lower than in other types of AML, laying between 20 and 50 years [3]. If untreated, the median survival of APL is one week [4]. However, if treated early with the recommended induction therapy, consisting of ATRA and an anthracycline (idarubicin or daunorubicin) or ATRA and ATO, APL is a highly curable disease [5].

Despite the dramatic impact of ATRA and ATO in the treatment of APL, the causes of treatment failures during induction therapy include fatalities resulting from hemorrhage and infection as general complications of chemotherapeutic therapy. Moreover, the adverse drug reaction DS is a serious and 
specific complication that can result from ATRA treatment of APL. While approximately half of early APL deaths are attributable to hemorrhage due to coagulopathy, DS remains one of the most frequently cited causes of death [6]. Up to a quarter of APL patients will experience DS during their treatment, yet evidence on the pathophysiology, epidemiology, predictive factors, and prevention is still scarce $[1,7,8]$.

DS was first described by Frankel et al. in 1992 as a distinct symptom complex characterized primarily by fever and dyspnea in the absence of infection, occurring during the treatment of patients treated with ATRA for APL [9]. Other symptoms were peripheral oedema, rapid weight gain, unexplained episodic hypotension, renal insufficiency, and hyperbilirubinemia. Frankel et al. noted that the syndrome only correlated with leukocytosis in some but not all patients and that the administration of intravenous dexamethasone brought rapid relief in most patients. While the authors named the symptom complex "retinoic acid syndrome" after the causative drug [9], "differentiation syndrome" is more commonly used in recent years [10,11].

Although DS is rare within the overall population, it occurs in up to one-quarter of patients treated with the differentiating agents, ATRA and ATO, making it a highly significant adverse drug reaction in these patients [7]. More recently, two clinical trials of isocitrate dehydrogenase (IDH)-inhibitor drugs for the treatment of relapsed/refractory IDH2-mutated (non-M3) AML unexpectedly observed DS as an adverse event, thereby identifying the IDH-inhibitors as potential differentiating agents [12-15]. The IDH-inhibitors enasidenib (IDHIFA ${ }^{\circledR}$, Celgene) and ivosidenib (TIBSOVO ${ }^{\circledR}$, Agios Pharmaceuticals) were approved by the US Food and Drug Administration (FDA) in 2017 and 2019, respectively. Due to the unexpected findings, the FDA included a boxed warning for the potential of DS with enasidenib in 2018 [16-19]. Additionally, the marketing authorization application of enasidenib submitted to the European Medicines Agency (EMA) was withdrawn by Celgene in December of 2019 due to a lack of a positive benefit/risk ratio in the proposed indication [20].

As there is limited knowledge of DS incidence, particularly among the non-M3 AML, we aimed to complete a systematic review on the incidence of DS with all treatment regimens in AML patients. In particular, we focused on the new IDH-inhibitor drugs compared to existing differentiating therapies based on ATRA and ATO to contextualize the occurrence of DS in these new agents.

\section{Methods}

\subsection{Literature Search and Article Selection}

We identified all studies published from 1 January 1992 through to 25 June 2019. The start date corresponds to the first report of DS published by Frankel et al. in 1992 [9]. We searched the PubMed database using the search string ("differentiation syndrome"[tw]) OR (different syndrom*[tw]) OR ("retinoic acid syndrome"[tw]) OR (retin acid syndrom*[tw]) AND (Humans[Mesh]). Articles were excluded if they were not published in English if they did not contain any information on the administered drug, and/or if they contained no, or insufficient, information on the occurrence of DS. We further excluded abstracts, commentaries, letters to the editor, and review articles. While we took all efforts to retrieve the full-text of all articles selected during the screening of title and abstract, we excluded articles that were not available for full-text review.

Screening of the articles was repeated independently by two authors (LG, LH), with disagreement resolved through discussion or consultation with a third author (AMB). While we identified all relevant publications, only clinical trials were eligible for detailed review. Furthermore, one author (LG) conducted a hand search of the reference lists among the selected articles retrieved from PubMed. Additionally, all included articles were cross-referenced with a retraction database to ensure none of the articles had retraction notices [21].

\subsection{Data Extraction}

The authors used a standardized data extraction format. The data extraction was performed per trial, combining information from multiple publications in one trial record, if necessary. Extracted 
information included patient demographics and information on the study treatment (administered drugs, doses, route, frequency, and duration). Furthermore, we extracted the number of patients who had experienced DS and the number of patients who died. If available, we extracted the time to onset of DS, as well as the treatment of DS. Clinical trial treatment arms were grouped according to their treatment regimen into "ATRA only," "ATO only," "ATRA + ATO," "ATRA + cytotoxic chemotherapy" ("ATRA + CT"), "IDH-inhibitors," and "retinoic acid derivatives." We categorized regimens that did not fit the above categories as "other."

\subsection{Risk of Bias Assessment}

We assessed all included clinical trials for the risk of bias using a modified version 2 of the Cochrane Risk of Bias Tool for randomized trials (RoB 2) [22]. The risk of bias assessment was conducted by the primary author (LG) for the reported primary outcome of the respective clinical trial and aimed to estimate the risk of bias on adhering to the intervention. We used information from the published journal article(s). However, when available, we used additional sources, such as clinical trial databases. We then assessed the level of bias that was likely (high) or unlikely (low) to impact the study results. The robvis package for $\mathrm{R}$ (3.5.2) generated the summary figures of the risk of bias assessment.

\subsection{Statistical Analysis}

We calculated the incidence of DS as a percentage of the whole study population. Additionally, we calculated the weighted mean cumulative incidence of DS in percent and the corresponding 95\% confidence intervals $(95 \% \mathrm{CI})$ within each treatment regimen. For the calculations of the cumulative incidence of DS, we considered only patients who had received a differentiating agent as part of their treatment regimen. Patients in treatment arms, not including a differentiating agent, were excluded from the calculation of the cumulative incidence of DS. We stratified results by the individual treatment arms (ATRA + ATO, ATRA + CT, ATRA, ATO, IDH-inhibitors, retinoic acid derivatives, other).

\section{Results}

We identified a total of 838 articles from PubMed; after removing duplicates and non-English studies, 591 titles and abstracts were screened, resulting in the exclusion of 434 articles (Figure 1). In addition to the remaining 157 full-text articles, we further identified 57 articles [12,23-78] from the hand-search of the reference lists, resulting in 214 full-text articles. Of these, we excluded 80 . Of the 134 eligible publications, 44 were clinical trial reports and included in the final analysis $[1,12,13,27$, $29,31,34,40,43,44,48,49,51-57,64,68,70,72,74-77,79-95]$. The remaining 90 were case reports $(n=67)$ or observational study reports $(n=23)$.

Characteristics of the 44 included publications, reporting on 39 unique clinical trials, are summarized in Table 1 and Supplementary Table S1. In the 39 clinical trials included in the systematic review, we identified a total of 6949 patients. Sex distribution was almost equal with $51.5 \%$ male $(n=3577)$ and $47.9 \%$ female $(n=3327)$ patients $(0.6 \%$ unknown, $n=45)$. The median ages ranged from 8 to 70 years in all 39 clinical trials. The three trials conducted in patients with non-M3 AML had reported median ages of 68,70 , and 69 , respectively $[12,13,93]$. The highest median age in the clinical trials, including only APL patients, was 65 [84]. 
Table 1. Overview of included clinical trials $(n=39)$ and patient characteristics, treatment regimens, and outcomes.

\begin{tabular}{|c|c|c|c|c|c|c|c|c|c|c|c|c|}
\hline \multirow{2}{*}{$\begin{array}{c}\text { Clinical Trials } \\
\text { Reference }\end{array}$} & \multicolumn{4}{|c|}{ Patients } & \multicolumn{4}{|c|}{ Treatment } & \multirow{2}{*}{$\%$ DS } & \multirow{2}{*}{$\begin{array}{c}\% \\
\text { Deaths }\end{array}$} & \multirow{2}{*}{$\begin{array}{c}\text { T.T.O. } \\
\text { [d] }\end{array}$} & \multirow{2}{*}{ (Range) } \\
\hline & Total & Age & (Range) & $\%$ Male & Allocation & & Primary Treatm & & & & & \\
\hline \multirow{4}{*}{ Zhu, 2013 [53] } & 231 & 37 & $(15-60)$ & 54.5 & & & & & 22.1 & 0 & & \\
\hline & 117 & 39 & $(15-60)$ & 55.6 & randomized & $\begin{array}{c}\text { ATRA } \\
25 \mathrm{mg} / \mathrm{m}^{2}\end{array}$ & $\begin{array}{c}\text { ATO } \\
0.16 \mathrm{mg} / \mathrm{kg}\end{array}$ & & 24.8 & 0 & & \\
\hline & 114 & 33 & $(15-60)$ & 53.5 & randomized & $\begin{array}{c}\text { ATRA } \\
25 \mathrm{mg} / \mathrm{m}^{2}\end{array}$ & $\begin{array}{c}\mathrm{RIF} \\
60 \mathrm{mg} / \mathrm{kg}\end{array}$ & & 19.3 & 0 & & \\
\hline & 124 & 44 & $(3-78)$ & 50.0 & & & & & 13.7 & 0 & & \\
\hline \multirow[t]{3}{*}{ Iland, 2012 [87] } & 108 & & & & $\leq 60 \mathrm{y}$. & $\begin{array}{c}\text { ATRA } \\
45 \mathrm{mg} / \mathrm{m}^{2} / \mathrm{d} \text { tid }\end{array}$ & $\begin{array}{c}\text { ATO } \\
0.15 \mathrm{mg} / \mathrm{kg} / \mathrm{d} \text { qd d9-36 }\end{array}$ & $\begin{array}{c}\text { idarubicin } \\
12 \mathrm{mg} / \mathrm{m}^{2} / \mathrm{d} \\
\mathrm{d} 2,4,6,8\end{array}$ & & & & \\
\hline & 9 & & & & $61-70 \mathrm{y}$ & $\begin{array}{c}\text { ATRA } \\
45 \mathrm{mg} / \mathrm{m}^{2} / \mathrm{d} \text { tid }\end{array}$ & $\begin{array}{c}\text { ATO } \\
0.15 \mathrm{mg} / \mathrm{kg} / \mathrm{d} \text { qd d9-36 }\end{array}$ & $\begin{array}{c}\text { idarubicin } \\
9 \mathrm{mg} / \mathrm{m}^{2} / \mathrm{d} \mathrm{d} 2,4,6,8\end{array}$ & & & & \\
\hline & 7 & & & & $>70 \mathrm{y}$ & $\begin{array}{c}\text { ATRA } \\
45 \mathrm{mg} / \mathrm{m}^{2} / \mathrm{d} \text { tid }\end{array}$ & $\begin{array}{c}\text { ATO } \\
0.15 \mathrm{mg} / \mathrm{kg} / \mathrm{d} \mathrm{qd} \mathrm{d9-36}\end{array}$ & $\begin{array}{c}\text { idarubicin } \\
6 \mathrm{mg} / \mathrm{m}^{2} / \mathrm{d} \mathrm{d} 2,4,6,8\end{array}$ & & & & \\
\hline \multirow{5}{*}{$\begin{array}{c}\text { Abaza, 2017 [49]; } \\
\text { Ravandi, } 2009 \\
\text { [55] }\end{array}$} & 187 & 50 & $(14-84)$ & 51.9 & & & & & 11.2 & 0 & & \\
\hline & & & & & $\mathrm{WBC}<10 \times 10^{9 / 1}$ old & $\begin{array}{c}\text { ATRA } \\
45 \mathrm{mg} / \mathrm{m}^{2} / \mathrm{d} \text { bid }\end{array}$ & $\begin{array}{c}\text { ATO } \\
0.15 \mathrm{mg} / \mathrm{kg} / \mathrm{d} \text { qd from } \\
\mathrm{d} 10\end{array}$ & & & & & \\
\hline & & & & & $\mathrm{WBC} \geq 10 \times 10^{9 / 1}$ old & $\begin{array}{c}\text { ATRA } \\
45 \mathrm{mg} / \mathrm{m}^{2} / \mathrm{d} \text { bid }\end{array}$ & $\begin{array}{c}\text { ATO } \\
0.15 \mathrm{mg} / \mathrm{kg} / \mathrm{d} \text { qd from } \\
\mathrm{d} 10\end{array}$ & $\begin{array}{c}\text { Gemtuzumab } \\
\text { ozogamicin } \\
9 \mathrm{mg} / \mathrm{m}^{2} / \mathrm{d} \text { qd d } 1\end{array}$ & & & & \\
\hline & & & & & $\mathrm{WBC}<10 \times 10^{9 / 1}$ new & $\begin{array}{c}\text { ATRA } \\
45 \mathrm{mg} / \mathrm{m}^{2} / \mathrm{d} \text { bid }\end{array}$ & $\begin{array}{c}\text { ATO } \\
0.15 \mathrm{mg} / \mathrm{kg} / \mathrm{d} \text { qd from d1 }\end{array}$ & & & & & \\
\hline & & & & & $\mathrm{WBC} \geq 10 \times 10^{9 / 1}$ new & $\begin{array}{c}\text { ATRA } \\
45 \mathrm{mg} / \mathrm{m}^{2} / \mathrm{d} \text { bid }\end{array}$ & $\begin{array}{c}\text { ATO } \\
0.15 \mathrm{mg} / \mathrm{kg} / \mathrm{d} \text { qd from d } 1\end{array}$ & $\begin{array}{l}\text { Gemtuzumab } \\
\text { ozogamicin } \\
9 \mathrm{mg} / \mathrm{m}^{2} / \mathrm{d} \text { qd d1 }\end{array}$ & & & & \\
\hline \multirow{3}{*}{ Dai, 2009 [54] } & 162 & & & 57.4 & & & & & 3.1 & 0 & & \\
\hline & 72 & 34 & $(14-69)$ & 54.2 & & $\begin{array}{c}\text { ATRA } \\
45 \mathrm{mg} / \mathrm{m}^{2} / \mathrm{d}\end{array}$ & & & 2.8 & & & \\
\hline & 90 & 32 & $(14-67)$ & 60.0 & & $\begin{array}{c}\text { ATRA } \\
45 \mathrm{mg} / \mathrm{m}^{2} / \mathrm{d}\end{array}$ & $\begin{array}{c}\text { ATO } \\
10 \mathrm{mg} / \mathrm{d} \text { d1-28 }\end{array}$ & & 3.3 & & & \\
\hline \multirow{3}{*}{$\begin{array}{c}\text { Platzbecker, } \\
2016 \text { [51]; } \\
\text { LoCoco, 2013 } \\
\text { [52] }\end{array}$} & 266 & & & 48.9 & & & & & 14.3 & 0.8 & & \\
\hline & 129 & 46.6 & $(19-70)$ & 46.5 & randomized & $\begin{array}{c}\text { ATRA } \\
45 \mathrm{mg} / \mathrm{m}^{2} / \mathrm{d} \text { bid }\end{array}$ & $\begin{array}{c}\text { ATO } \\
0.15 \mathrm{mg} / \mathrm{kg} / \mathrm{d} \mathrm{gd}\end{array}$ & & 16.3 & 0 & & \\
\hline & 137 & 46.6 & $(18-70)$ & 51.1 & randomized & $\begin{array}{c}\text { ATRA } \\
45 \mathrm{mg} / \mathrm{m}^{2} / \mathrm{d} \text { bid }\end{array}$ & $\begin{array}{c}\text { idarubicin } \\
12 \mathrm{mg} / \mathrm{m}^{2} / \mathrm{d} \mathrm{qd} \mathrm{d} 2,4,6,8\end{array}$ & & 12.4 & 1.5 & & \\
\hline
\end{tabular}


Table 1. Cont.

\begin{tabular}{|c|c|c|c|c|c|c|c|c|c|c|c|c|c|}
\hline \multirow{2}{*}{$\begin{array}{c}\text { Clinical Trials } \\
\text { Reference }\end{array}$} & \multicolumn{4}{|c|}{ Patients } & \multicolumn{5}{|c|}{ Treatment } & \multirow{2}{*}{$\%$ DS } & \multirow{2}{*}{$\begin{array}{c}\% \\
\text { Deaths }\end{array}$} & \multirow{2}{*}{$\begin{array}{l}\text { T.T.O. } \\
\text { [d] }\end{array}$} & \multirow{2}{*}{ (Range) } \\
\hline & Total & Age & (Range) & $\%$ Male & Allocation & & Primary Treatm & & & & & & \\
\hline \multirow{3}{*}{ Yang, 2018 [40] } & 82 & 9.4 & $(1-16)$ & 62.2 & & & & & & 6.1 & & & \\
\hline & 42 & 7.8 & $(1-13)$ & 69.0 & randomized & $\begin{array}{c}\text { ATRA } \\
25 \mathrm{mg} / \mathrm{m}^{2} / \mathrm{d}\end{array}$ & $\begin{array}{c}\text { mitoxantrone } \\
10 \text { or } 7 \mathrm{mg} / \mathrm{m}^{2} / \mathrm{d} \mathrm{d} 3 \text { or } \\
\mathrm{d} 2-4\end{array}$ & $\begin{array}{c}\mathrm{ATO} \\
0.16 \mathrm{mg} / \mathrm{kg} / \mathrm{d} \mathrm{qd} \\
\text { from d5/6 }\end{array}$ & & 9.5 & & & \\
\hline & 40 & 9.9 & $(2-16)$ & 55.0 & randomized & $\begin{array}{c}\text { ATRA } \\
25 \mathrm{mg} / \mathrm{m}^{2} / \mathrm{d}\end{array}$ & $\begin{array}{c}\text { mitoxantrone } \\
10 \text { or } 7 \mathrm{mg} / \mathrm{m}^{2} / \mathrm{d} \mathrm{d} 3 \text { or } \\
\mathrm{d} 2-4\end{array}$ & $\begin{array}{c}\text { RIF1 } \\
35 \mathrm{mg} / \mathrm{kg} / \mathrm{d} \text { tid } \\
\text { from d5/6 }\end{array}$ & & 2.5 & & & \\
\hline \multirow{3}{*}{$\begin{array}{c}\text { Burnett, } 2015 \\
\text { [77] }\end{array}$} & 235 & 47 & $(16-77)$ & 51.1 & & & & & & 23.4 & 0 & & \\
\hline & 119 & 47 & $(16-77)$ & 50.4 & randomized & $\begin{array}{c}\text { ATRA } \\
45 \mathrm{mg} / \mathrm{m}^{2} / \mathrm{d} \text { bid }\end{array}$ & $\begin{array}{c}\text { idarubicin } \\
12 \mathrm{mg} / \mathrm{m}^{2} / \mathrm{d} \mathrm{qd} \mathrm{d} 2,4,6,8\end{array}$ & & & 21.0 & & & \\
\hline & 116 & 47 & $(16-75)$ & 51.7 & randomized & $\begin{array}{c}\text { ATRA } \\
45 \mathrm{mg} / \mathrm{m}^{2} / \mathrm{d} \text { bid }\end{array}$ & $\begin{array}{c}\text { ATO } \\
0.3 \mathrm{mg} / \mathrm{kg} / \mathrm{d} \mathrm{qd} \mathrm{d} 1-5 \& \\
\text { wk2-8 }\end{array}$ & & & 25.9 & & & \\
\hline$\underset{\text { [92] }}{\text { Imaizumi, } 2010}$ & 58 & 11 & $(1-16)$ & 53.4 & & $\begin{array}{c}\text { ATRA } \\
45 \mathrm{mg} / \mathrm{m}^{2} / \mathrm{d}\end{array}$ & $\begin{array}{c}\text { daunorubicin } \\
45 \mathrm{mg} / \mathrm{m}^{2} / \mathrm{d} \mathrm{d} 6-8\end{array}$ & $\begin{array}{c}\text { cytarabine } \\
200 \mathrm{mg} / \mathrm{m}^{2} / \mathrm{d} \\
\mathrm{d} 6-12\end{array}$ & & 7.3 & 0 & & \\
\hline \multirow{4}{*}{ Sanz, 2010 [44] } & 402 & 42 & (3-83) & 52.0 & & & & & & 28.5 & 1.1 & & \\
\hline & & & & & $20-70 \mathrm{y}$. & $\begin{array}{c}\text { ATRA } \\
45 \mathrm{mg} / \mathrm{m}^{2} / \mathrm{d} \text { bid }\end{array}$ & $\begin{array}{c}\text { idarubicin } \\
12 \mathrm{mg} / \mathrm{m}^{2} / \mathrm{d} \mathrm{gd} \mathrm{d} 2,4,6,8\end{array}$ & & & & & & \\
\hline & 22 & & & & $>70 \mathrm{y}$ & $\begin{array}{c}\text { ATRA } \\
45 \mathrm{mo} / \mathrm{m}^{2} / \mathrm{d} \text { bid }\end{array}$ & $\begin{array}{l}\text { idarubicin } \\
\text { id }\end{array}$ & & & & & & \\
\hline & & & & & $<20 \mathrm{y}$ & $\begin{array}{c}\text { ATRA } \\
25 \mathrm{mg} / \mathrm{m}^{2} / \mathrm{d} \text { bid }\end{array}$ & $\begin{array}{l}\text { idarubicin } \\
12 \mathrm{mg} / \mathrm{m}^{2} / \mathrm{d} \mathrm{qd} \mathrm{d} 2,4,6,8\end{array}$ & & & & & & \\
\hline \multirow[t]{2}{*}{$\begin{array}{c}\text { Lengfelder, } 2009 \\
\text { [74] }\end{array}$} & 142 & 40 & $(16-60)$ & 41.5 & 1st induction cycle & $\begin{array}{c}\text { ATRA } \\
45 \mathrm{mg} / \mathrm{m}^{2} / \mathrm{d}\end{array}$ & $\begin{array}{l}\text { 6-thioguanine } \\
100 \mathrm{mg} / \mathrm{m}^{2} \text { bid d3-9 }\end{array}$ & $\begin{array}{c}\text { cytarabine } \\
100 \mathrm{mg} / \mathrm{m}^{2} \mathrm{~d} 1-2 \\
\text { bid d3-8 }\end{array}$ & $\begin{array}{c}\text { daunorubicin } \\
60 \\
\mathrm{mg} / \mathrm{m}^{2} \\
\mathrm{~d} 3-5\end{array}$ & 21.1 & 0.8 & & \\
\hline & 131 & & & & 2nd induction cycle & $\begin{array}{c}\text { cytarabine } \\
3 \mathrm{~g} / \mathrm{m}^{2} \text { bid d21-23 }\end{array}$ & $\begin{array}{c}\text { mitoxantrone } \\
10 \mathrm{mg} / \mathrm{m}^{2} \mathrm{~d} 23-25\end{array}$ & & & & & & \\
\hline \multirow{3}{*}{$\begin{array}{c}\text { De Botton, } 2003 \\
\text { [57] }\end{array}$} & 306 & & & 44.1 & & & & & & 12.7 & 1.3 & & \\
\hline & 122 & 45.5 & $(35-54)$ & 45.9 & randomized & $\begin{array}{c}\text { ATRA } \\
45 \mathrm{mg} / \mathrm{m}^{2} / \mathrm{d}\end{array}$ & & & & 18.0 & 2.5 & 10 & \\
\hline & 184 & 45 & $(34-55)$ & 42.9 & randomized & $\begin{array}{c}\text { ATRA } \\
45 \mathrm{mg} / \mathrm{m}^{2} / \mathrm{d}\end{array}$ & $\begin{array}{c}\text { daunorubicin } \\
60 \mathrm{mg} / \mathrm{kg} / \mathrm{d} \mathrm{d} 3-5\end{array}$ & $\begin{array}{c}\text { cytarabine } \\
200 \mathrm{mg} / \mathrm{m}^{2} / \mathrm{d} \text { d3-9 }\end{array}$ & & 9.2 & 0.5 & 10.5 & \\
\hline \multirow{5}{*}{$\begin{array}{l}\text { Montesinos, } \\
2009[1]\end{array}$} & 739 & 40 & $(2-83)$ & 50.6 & & & & & & 24.8 & 1.4 & 12 & $(0-46)$ \\
\hline & & & & & $20-70 \mathrm{y}$. & ATRA & idarubicin & & & & & & \\
\hline & & & & & & $\begin{array}{c}45 \mathrm{mg} / \mathrm{m}^{2} / \mathrm{d} \text { bid } \\
\text { ATRA }\end{array}$ & $\begin{array}{c}12 \mathrm{mg} / \mathrm{m}^{2} / \mathrm{d} \mathrm{qd} \mathrm{d2,4,6,8} \\
\text { idarubicin }\end{array}$ & & & & & & \\
\hline & & & & & $>70 \mathrm{y}$. & $45 \mathrm{mg} / \mathrm{m}^{2} / \mathrm{d}$ bid & $12 \mathrm{mg} / \mathrm{m}^{2} / \mathrm{d}$ qd d2,4,6 & & & & & & \\
\hline & & & & & $<20 \mathrm{y}$ & $\begin{array}{c}\text { ATRA } \\
25 \mathrm{mg} / \mathrm{m}^{2} / \mathrm{d} \text { bid }\end{array}$ & $\begin{array}{c}\text { idarubicin } \\
12 \mathrm{mg} / \mathrm{m}^{2} / \mathrm{d} \mathrm{gd} \mathrm{d} 2,4,6,8\end{array}$ & & & & & & \\
\hline
\end{tabular}


Table 1. Cont

\begin{tabular}{|c|c|c|c|c|c|c|c|c|c|c|c|c|}
\hline \multirow{2}{*}{$\begin{array}{c}\text { Clinical Trials } \\
\text { Reference }\end{array}$} & \multicolumn{4}{|c|}{ Patients } & \multicolumn{4}{|c|}{ Treatment } & \multirow{2}{*}{$\%$ DS } & \multirow{2}{*}{$\begin{array}{c}\% \\
\text { Deaths }\end{array}$} & \multirow{2}{*}{$\begin{array}{l}\text { T.T.O. } \\
\text { [d] }\end{array}$} & \multirow{2}{*}{ (Range) } \\
\hline & Total & Age & (Range) & $\%$ Male & Allocation & & Primary Treatn & & & & & \\
\hline \multirow{3}{*}{$\begin{array}{l}\text { LoCoco, 2010 } \\
\quad[70] ; \\
\text { Testi 2005 [56] }\end{array}$} & 752 & & & 53.7 & & & & & 11.3 & 0.1 & & \\
\hline & 642 & 38.2 & $(18-61)$ & 54.4 & $\geq 18 \mathrm{y}$ & $\begin{array}{c}\text { ATRA } \\
45 \mathrm{mg} / \mathrm{m}^{2} / \mathrm{d}\end{array}$ & $\begin{array}{c}\text { idarubicin } \\
12 \mathrm{mg} / \mathrm{m}^{2} / \mathrm{d} \mathrm{qd} \mathrm{d2,4,6,8}\end{array}$ & & 12.9 & 0.2 & & \\
\hline & 110 & 11.6 & $(1-18)$ & 50.0 & $<18 \mathrm{y}$ & $\begin{array}{c}\text { ATRA } \\
25 \mathrm{mg} / \mathrm{m}^{2} / \mathrm{d} \\
\end{array}$ & $\begin{array}{c}\text { idarubicin } \\
12 \mathrm{mg} / \mathrm{m}^{2} / \mathrm{d} \text { qd d2,4,6,8 }\end{array}$ & & 1.8 & 0 & 7.5 & $(4-11)$ \\
\hline $\begin{array}{l}\text { LoCoco, } 2010 \\
\text { [70] }\end{array}$ & 453 & 40.9 & $(18-61)$ & 50.6 & & $\begin{array}{c}\text { ATRA } \\
45 \mathrm{mg} / \mathrm{m}^{2} / \mathrm{d}\end{array}$ & $\begin{array}{c}\text { idarubicin } \\
12 \mathrm{mg} / \mathrm{m}^{2} / \mathrm{d} \text { qd d2,4,6,8 }\end{array}$ & & 10.3 & 0.2 & & \\
\hline \multirow{3}{*}{$\begin{array}{c}\text { Colovic, } 1997 \\
\quad[90]\end{array}$} & 30 & & & 40.0 & & & & & 13.3 & 13.3 & & \\
\hline & 15 & 40 & $(16-65)$ & 26.7 & $\begin{array}{c}\text { WBC } \\
<5 \times 10^{9 / 1}\end{array}$ & $\begin{array}{c}\text { ATRA } \\
45 \mathrm{mg} / \mathrm{m}^{2} / \mathrm{d} \text { bid }\end{array}$ & & & 6.7 & 6.7 & & \\
\hline & 15 & 40 & $(18-60)$ & 53.3 & $\mathrm{WBC}>5 \times 10^{9 / 1}$ & $\begin{array}{c}\text { ATRA } \\
45 \mathrm{mg} / \mathrm{m}^{2} / \mathrm{d} \text { bid }\end{array}$ & $\begin{array}{c}\text { daunorubicin } \\
50 \mathrm{mg} / \mathrm{m}^{2} / \mathrm{d} 3 \mathrm{~d}\end{array}$ & $\begin{array}{c}\text { cytarabine } \\
200 \mathrm{mg} / \mathrm{m}^{2} / \mathrm{d} 7 \mathrm{~d}\end{array}$ & 20.0 & 20 & & \\
\hline \multirow{3}{*}{$\begin{array}{c}\text { Fenaux, } 1993 \\
\quad[43]\end{array}$} & 101 & 40 & $(6-67)$ & 52.5 & & & & & & & & \\
\hline & 54 & 41.5 & $(6-63)$ & 55.6 & randomized & $\begin{array}{c}\text { ATRA } \\
45 \mathrm{mg} / \mathrm{m}^{2} / \mathrm{d}\end{array}$ & $\begin{array}{l}\text { daunorubicin } \\
60 \mathrm{mg} / \mathrm{m}^{2} / \mathrm{d} 3 \mathrm{~d}\end{array}$ & $\begin{array}{c}\text { cytarabine } \\
200 \mathrm{mg} / \mathrm{m}^{2} / \mathrm{d} 7 \mathrm{~d}\end{array}$ & 5.6 & 0 & 20 & $(14-24)$ \\
\hline & 47 & 40 & $(17-67)$ & 48.9 & randomized & & $\begin{array}{c}\text { daunorubicin } \\
60 \mathrm{mg} / \mathrm{m}^{2} / \mathrm{d} 2 \times 3 \mathrm{~d}\end{array}$ & $\begin{array}{c}\text { cytarabine } \\
200 \mathrm{mg} / \mathrm{m}^{2} / \mathrm{d} 2 \times \\
7 \mathrm{~d}\end{array}$ & & & & \\
\hline Powell, 2010 [31] & 481 & & $(15-79)$ & 51.4 & & $\begin{array}{c}\text { ATRA } \\
45 \mathrm{mg} / \mathrm{m}^{2} / \mathrm{d} \text { bid }\end{array}$ & $\begin{array}{c}\text { daunorubicin } \\
50 \mathrm{mg} / \mathrm{m}^{2} / \mathrm{d} \text { qd d3-6 }\end{array}$ & $\begin{array}{c}\text { cytarabine } \\
200 \mathrm{mg} / \mathrm{m}^{2} / \mathrm{d} \text { d3-9 }\end{array}$ & 36.8 & & & \\
\hline $\begin{array}{c}\text { Frankel, } 1994 \\
{[81]}\end{array}$ & 56 & & $(9-75)$ & 44.6 & & $\begin{array}{c}\text { ATRA } \\
45 \mathrm{mg} / \mathrm{m}^{2} / \mathrm{d} \text { bid }\end{array}$ & & & 23.2 & 8.9 & & \\
\hline \multirow{3}{*}{$\begin{array}{c}\text { Tallman, } 1997 \\
\text { [68]; } \\
\text { Tallman, } 2000 \\
\text { [82] }\end{array}$} & 346 & & & 51.7 & & & & & & & & \\
\hline & 172 & 37 & $(1-81)$ & 47.7 & randomized & $\begin{array}{c}\text { ATRA } \\
45 \mathrm{mg} / \mathrm{m}^{2} / \mathrm{d} \text { bid }\end{array}$ & & & 26.3 & 1.2 & 11 & $(2-47)$ \\
\hline & 174 & 38 & $(1-74)$ & 55.7 & & $\begin{array}{c}\text { daunorubicin } \\
45 \mathrm{mg} / \mathrm{m}^{2} / \mathrm{d} \text { qd } \\
\mathrm{d} 1-36\end{array}$ & $\begin{array}{c}\text { cytarabine } \\
100 \mathrm{mg} / \mathrm{m}^{2} / \mathrm{d} \mathrm{d} 1-7\end{array}$ & & & & & \\
\hline $\begin{array}{c}\text { Castaigne, } 1993 \\
\text { [89] }\end{array}$ & 30 & 56 & $(10-81)$ & 43.3 & & $\begin{array}{c}\text { ATRA } \\
25 \mathrm{mg} / \mathrm{m}^{2} / \mathrm{d} \text { bid }\end{array}$ & & & 6.7 & 6.7 & & \\
\hline Douer, 2001 [77] & 69 & 44 & $(5-82)$ & 58.0 & & $\begin{array}{c}\text { liposomal ATRA } \\
90 \mathrm{mg} / \mathrm{m}^{2} \mathrm{qad}\end{array}$ & & & 26.1 & 1.4 & & \\
\hline $\begin{array}{c}\text { Advani, } 1999 \\
\text { [94] }\end{array}$ & 43 & & $(7-60)$ & 65.1 & & $\begin{array}{c}\text { ATRA } \\
45 \mathrm{mg} / \mathrm{m}^{2} / \mathrm{d}\end{array}$ & & & 32.6 & 14.0 & 10 & $(4-26)$ \\
\hline $\begin{array}{l}\text { de Medeiros, } \\
1998 \text { [79] }\end{array}$ & 37 & 17.5 & $(9-69)$ & 45.9 & & $\begin{array}{c}\text { ATRA } \\
45 \mathrm{mg} / \mathrm{m}^{2} / \mathrm{d}\end{array}$ & & & 10.8 & 0 & & \\
\hline
\end{tabular}


Table 1. Cont

\begin{tabular}{|c|c|c|c|c|c|c|c|c|c|c|c|}
\hline \multirow{2}{*}{$\begin{array}{c}\text { Clinical Trials } \\
\text { Reference }\end{array}$} & \multicolumn{4}{|c|}{ Patients } & \multicolumn{3}{|r|}{ Treatment } & \multirow{2}{*}{$\%$ DS } & \multirow{2}{*}{$\begin{array}{c}\% \\
\text { Deaths }\end{array}$} & \multirow{2}{*}{$\begin{array}{l}\text { T.T.O. } \\
\text { [d] }\end{array}$} & \multirow{2}{*}{ (Range) } \\
\hline & Total & Age & (Range) & $\%$ Male & Allocation & & Primary Treatment & & & & \\
\hline $\begin{array}{c}\text { Mandegary, } 2010 \\
{[86]}\end{array}$ & 20 & 31 & $(15-62)$ & 35.0 & & $\begin{array}{c}\mathrm{ATO} \\
15 \mathrm{mg} / \mathrm{kg} / \mathrm{d} \mathrm{qd}\end{array}$ & & 60.0 & 5.0 & & \\
\hline $\begin{array}{c}\text { Ghavamzadeh, } \\
2006[72]\end{array}$ & 111 & 27 & $(6-79)$ & 45.9 & & $\begin{array}{c}\mathrm{ATO} \\
15 \mathrm{mg} / \mathrm{kg} / \mathrm{d} \mathrm{qd}\end{array}$ & & 20.7 & 7.2 & & \\
\hline $\begin{array}{c}\text { Mathews, } 2006 \\
{[64]}\end{array}$ & 72 & 28 & $(3-75)$ & 52.8 & $\begin{array}{c}\text { adults } \\
\text { pediatric patients }\end{array}$ & $\begin{array}{c}\text { ATO } \\
10 \mathrm{mg} / \mathrm{d} \\
\text { ATO } \\
0.15 \mathrm{mg} / \mathrm{kg} / \mathrm{d}\end{array}$ & $\begin{array}{c}\text { hydroxyurea } \\
0-4 \mathrm{~g} / \mathrm{d} \\
\text { hydroxyurea } \\
0-30 \mathrm{mg} / \mathrm{kg} / \mathrm{d} \text { qd-qid }\end{array}$ & 6.9 & 0 & $13.2 *$ & $(6-21)$ \\
\hline Zhou, 2010 [27] & $\begin{array}{c}19 \\
5 \\
14\end{array}$ & 10 & $(4-15)$ & 57.9 & $\begin{array}{l}4-6 \mathrm{y} \\
>6 \mathrm{y} .\end{array}$ & $\begin{array}{c}\mathrm{ATO} \\
0.2 \mathrm{mg} / \mathrm{kg} / \mathrm{d} \mathrm{qd} \\
\mathrm{ATO} \\
0.16 \mathrm{mg} / \mathrm{kg} / \mathrm{d} \mathrm{qd}\end{array}$ & & 10.5 & 0 & & \\
\hline Hao, 2013 [95] & 46 & 8 & (mean) & 76.1 & & $\begin{array}{c}\text { ATO } \\
0.17-0.33 \mathrm{mg} / \mathrm{kg} / \mathrm{d} \\
\text { qd }\end{array}$ & & 17.4 & & & \\
\hline $\begin{array}{c}\text { Soignet, } 1998 \\
\text { [76] }\end{array}$ & 12 & 33.5 & $(9-75)$ & & & $\begin{array}{c}\text { ATO } \\
0.06-0.2 \mathrm{mg} / \mathrm{kg} / \mathrm{d}\end{array}$ & & 16.7 & 0 & & \\
\hline $\begin{array}{c}\text { Soignet, 2001 } \\
\text { [29] }\end{array}$ & 40 & & & 60.0 & & $\begin{array}{c}\mathrm{ATO} \\
0.15 \mathrm{mg} / \mathrm{kg} / \mathrm{d}\end{array}$ & & 25.0 & 0 & & \\
\hline Jin, 2006 [85] & 30 & & $(18-65)$ & 60.0 & & $\begin{array}{c}\text { ATO } \\
10 \mathrm{mg} \text { qd }\end{array}$ & & 30.0 & 0 & $13.9^{*}$ & $(5-25)$ \\
\hline $\begin{array}{c}\text { Shigeno, } 2005 \\
\text { [80]; } \\
\text { Ohnishi, 2002 } \\
{[83]} \\
\end{array}$ & 34 & 47 & $(17-82)$ & 64.7 & & $\begin{array}{c}\text { ATO } \\
0.15 \mathrm{mg} / \mathrm{kg} / \mathrm{d}\end{array}$ & & 23.5 & 0 & & \\
\hline $\begin{array}{c}\text { DiNardo, } 2018 \\
\quad[13]\end{array}$ & 258 & 68 & $(18-89)$ & 53.1 & dose-escalation & $\begin{array}{c}\text { ivosidenib } \\
100 \mathrm{mg} \\
\mathrm{bid} / 300-1200 \mathrm{mg} \\
\mathrm{qd} \\
\end{array}$ & & 11.2 & 0 & & \\
\hline $\begin{array}{l}\text { Stein, } 2017 \text { [91]; } \\
\text { Fathi, } 2018 \text { [12] }\end{array}$ & 239 & 70 & (19-100) & 57.3 & dose-escalation & $\begin{array}{c}\text { enasidenib } \\
30-150 \mathrm{mg} \\
\text { bid/50-650 mg qd }\end{array}$ & & 9.6 & & 48 & $(10-340)$ \\
\hline Tsai, 2008 [93] & 27 & 69 & $(51-82)$ & 70.4 & dose-escalation & $\begin{array}{c}\text { bexarotene } \\
100-300 \mathrm{mg} / \mathrm{m}^{2} \\
400 \mathrm{mg} / \mathrm{m}^{2}\end{array}$ & & 7.4 & 0 & & \\
\hline
\end{tabular}


Table 1. Cont.

\begin{tabular}{|c|c|c|c|c|c|c|c|c|c|c|c|c|}
\hline \multirow{2}{*}{$\begin{array}{c}\text { Clinical Trials } \\
\text { Reference }\end{array}$} & \multicolumn{4}{|c|}{ Patients } & \multicolumn{4}{|c|}{ Treatment } & \multirow{2}{*}{$\%$ DS } & \multirow{2}{*}{$\begin{array}{c}\% \\
\text { Deaths }\end{array}$} & \multirow{2}{*}{$\begin{array}{l}\text { T.T.O. } \\
\text { [d] }\end{array}$} & \multirow{2}{*}{ (Range) } \\
\hline & Total & Age & (Range) & $\%$ Male & Allocation & & Primary Treatr & & & & & \\
\hline Tobita, 1997 [88] & 24 & 49 & $(19-76)$ & 54.2 & & $\begin{array}{l}\text { tamibarotene } \\
6 \mathrm{mg} / \mathrm{m}^{2} / \mathrm{d} \text { bid }\end{array}$ & & & 4.2 & 0 & 18 & \\
\hline \multirow{3}{*}{ Zhang, 2013 [84] } & 33 & 65 & $(60-79)$ & & & & & & 15.2 & 0 & & \\
\hline & & & & & $\mathrm{WBC} \leq 20 \times 10^{9 / 1}$ & ATO & & & & & & \\
\hline & & & & & $\mathrm{WBC}>20 \times 10^{9 / 1}$ & $\begin{array}{c}\mathrm{ATO} \\
0.08 \mathrm{mg} / \mathrm{kg} / \mathrm{d} \text { qd }\end{array}$ & $\begin{array}{l}\text { daunorubicin } \\
40 \mathrm{mg} \mathrm{d} 1-3\end{array}$ & $\begin{array}{c}\text { cytarabine } \\
50-100 \mathrm{mg} \text { d1-5 }\end{array}$ & & & & \\
\hline \multirow{4}{*}{ Asou, 2007 [34] } & 283 & 48 & $(15-70)$ & 55.8 & & & & & 21.2 & 0.7 & & \\
\hline & 85 & & & & $\mathrm{WBC}<3 \times 10^{9 / 1}$ & $\begin{array}{c}\text { ATRA } \\
45 \mathrm{mg} / \mathrm{m}^{2} / \mathrm{d} \text { tid }\end{array}$ & & & & & & \\
\hline & 139 & & & & $\begin{array}{c}3 \times 10^{9 / 1} \leq \mathrm{WBC}<10 \\
\times 10^{9 / 1}\end{array}$ & $\begin{array}{c}\text { ATRA } \\
45 \mathrm{mg} / \mathrm{m}^{2} / \mathrm{d} \text { tid }\end{array}$ & $\begin{array}{c}\text { idarubicin } \\
12 \mathrm{mg} / \mathrm{m}^{2} / \mathrm{d} \text { qd d1-2 }\end{array}$ & $\begin{array}{c}\text { cytarabine } \\
80 \mathrm{mg} / \mathrm{m}^{2} / \mathrm{d} \text { d1-5 }\end{array}$ & & & & \\
\hline & 52 & & & & $\mathrm{WBC}>10 \times 10^{9 / 1}$ & $\begin{array}{c}\text { ATRA } \\
45 \mathrm{mg} / \mathrm{m}^{2} / \mathrm{d} \text { tid }\end{array}$ & $\begin{array}{l}\text { idarubicin } \\
12 \mathrm{mg} / \mathrm{m}^{2} / \mathrm{d} \text { qd d1-3 }\end{array}$ & $\begin{array}{c}\text { cytarabine } \\
100 \mathrm{mg} / \mathrm{m}^{2} / \mathrm{d} \text { d1-5 }\end{array}$ & & & & \\
\hline \multirow{3}{*}{ Asou, 2001 [75] } & 369 & 46 & $(15-85)$ & 46.9 & & & & & 7.6 & 0.3 & & \\
\hline & 126 & & & 54.5 & $\mathrm{WBC}<3 \times 10^{9 / 1}$ & $\begin{array}{c}\text { ATRA } \\
45 \mathrm{mg} / \mathrm{m}^{2} \text { tid }\end{array}$ & & & & & & \\
\hline & 243 & & & 55.6 & $\mathrm{WBC} \geq 3 \times 10^{9 / 1}$ & $\begin{array}{c}\text { ATRA } \\
45 \mathrm{mg} / \mathrm{m}^{2} \text { tid }\end{array}$ & $\begin{array}{c}\text { daunorubicin } \\
40 \mathrm{mg} / \mathrm{m}^{2} / \mathrm{d} \mathrm{qd} 3 \mathrm{~d}\end{array}$ & $\begin{array}{c}\text { enocitabine } \\
200 \mathrm{mg} / \mathrm{m}^{2} / \mathrm{d} \text { qd } \\
5 \mathrm{~d}\end{array}$ & & & & \\
\hline
\end{tabular}

Abbreviations: DS = Differentiation syndrome; TTO $=$ Time to treatment onset, ATRA: All trans retinoic acid, ATO $=$ arsenic trioxide, $\mathrm{mg}^{=}$milligrams, $\mathrm{m}^{2}=$ meters squared, $\mathrm{d}=$ day, tid, $\mathrm{kg}=$ kilograms, $\mathrm{qd}=$ once daily, bid = twice daily, tid = three times daily, qid = four times daily, qad = every other day; $\mathrm{d} 5=$ day 5 after start of treatment WBC = white blood cells. Variables: total = total number of patients in the clinical trial $(\mathrm{arm})$; age = median age in years; allocation = criterion for the assignment of patients to the respective clinical trial arm; Primary treatment $=$ describes drugs, doses, and frequencies of administration of the drugs administered for remission induction; \%DS = proportion of patients who experienced DS in the respective clinical trial (arm); \% deaths = proportion of patients who died as a consequence of DS; TTO = median (mean if marked with *) time to onset of DS after treatment start given in days. Notes: Information applicable to the whole clinical trial population is provided in the first row for each author. If individual trial arm data were available, the treatment-arm specific information is provided in the row's underneath (one arm per row). Induction regimen description the dosing information is provided beneath the corresponding drug name. If a field is empty in the table, the value was not stated in the publication. 


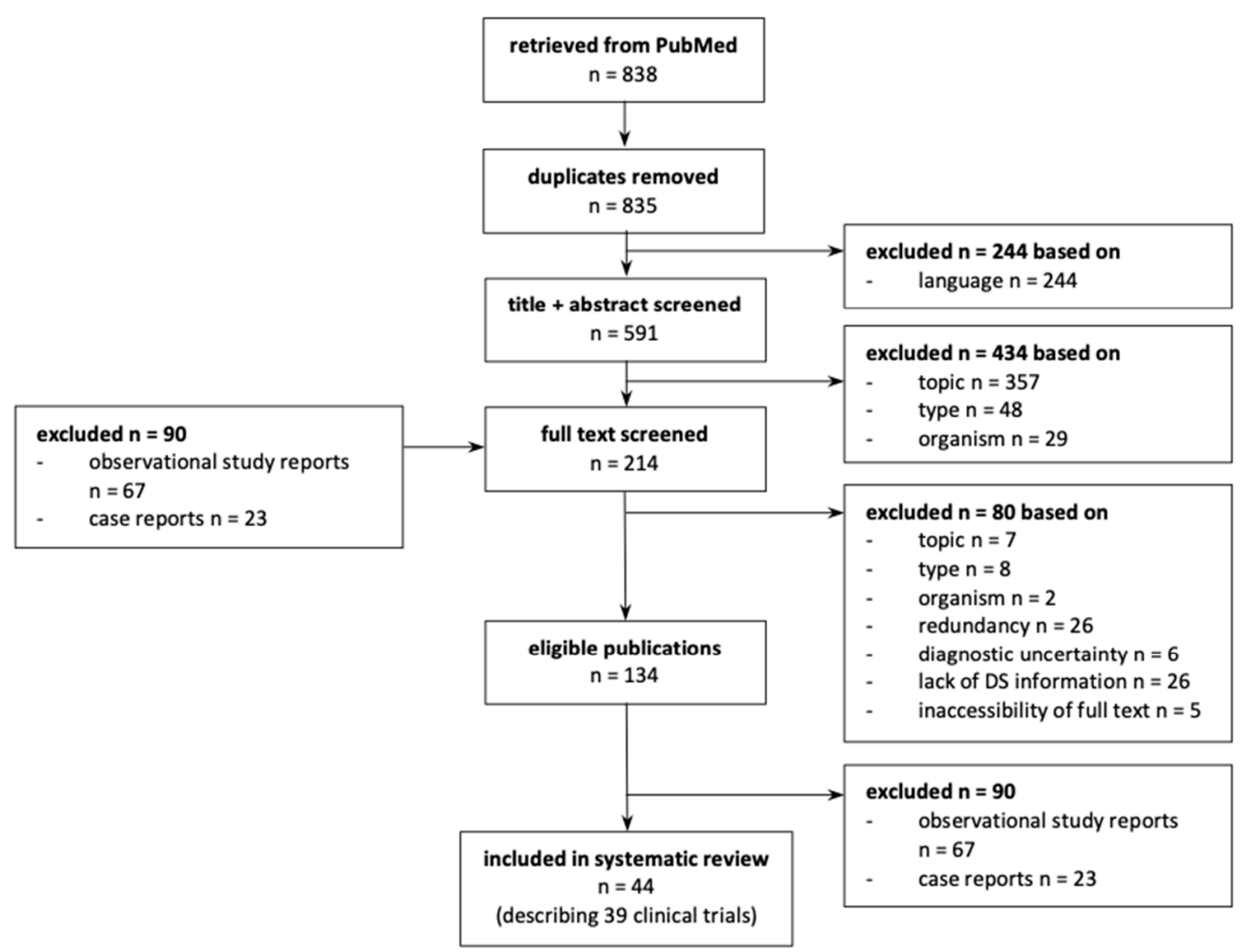

Figure 1. Article selection flow diagram. Redundant described a study when the original report was present, and the article did not add any further information. Of five articles, a full-text version was not available to our institution.

Of all 6728 patients in clinical trials treated with a differentiating agent, a mean of $17.7 \%$ (95\% CI 14.9-20.4) experienced DS. Of all patients in clinical trials treated with ATRA and ATO concomitantly $(n=959,14.3 \%)$, a weighted mean of $15.4 \%$ (95\% CI 10.2-20.7) experienced DS. If treated with ATRA and concomitant cytotoxic chemotherapy (ATRA + CT) $(n=3536,52.6 \%)$, DS occurred in a mean of $19.6 \%$ (95\% CI 14.2-25.0) of patients. Treatment with single-agent ATRA $(n=616,9.2 \%)$ or ATO $(n=384$, $5.7 \%$ ) resulted in cumulative DS incidences of $19.5 \%$ (95\% CI 13.7-25.3) and 20.6\% (95\% CI 12.9-28.2), respectively. In the clinical trials of the IDH-inhibitors ivosidenib and enasidenib, comprising a total of 497 patients (7.4\%), DS occurred in a mean of $10.4 \%$ (95\% CI 9.3-11.5) of patients. Trial treatment with the retinoic acid derivatives bexarotene and tamibarotene led to DS in a mean of 5.9\% (95\% CI 3.6-8.2) of the study population $(n=51)$.

Of the 39 clinical trials, three $(7.7 \%)$ had a low risk of bias, four had some concerns $(10.2 \%)$, and $32(82.1 \%)$ had a high risk of bias. Figure 2 illustrates the risk of bias by study and treatment group. The primary driver of the high risk of bias was the randomization process domain (Figure 3 ). It is to be noted that most of the included clinical trials were not randomized but instead used a risk- or age-based approach for assignment of the intervention or did not have a control arm, for example, the included phase 1 and 2 trials of ivosidenib and enasidenib. Recalculating the mean weighted incidence of DS from the clinical trials with a low risk of bias [40,51-53] or some concerns [31,34,43,68,82] only, 17.4\% of patients treated with ATRA + ATO, 29.3\% of patients treated with ATRA + CT, and 25.6\% of patients treated with single-agent ATRA experienced DS (results not shown). 


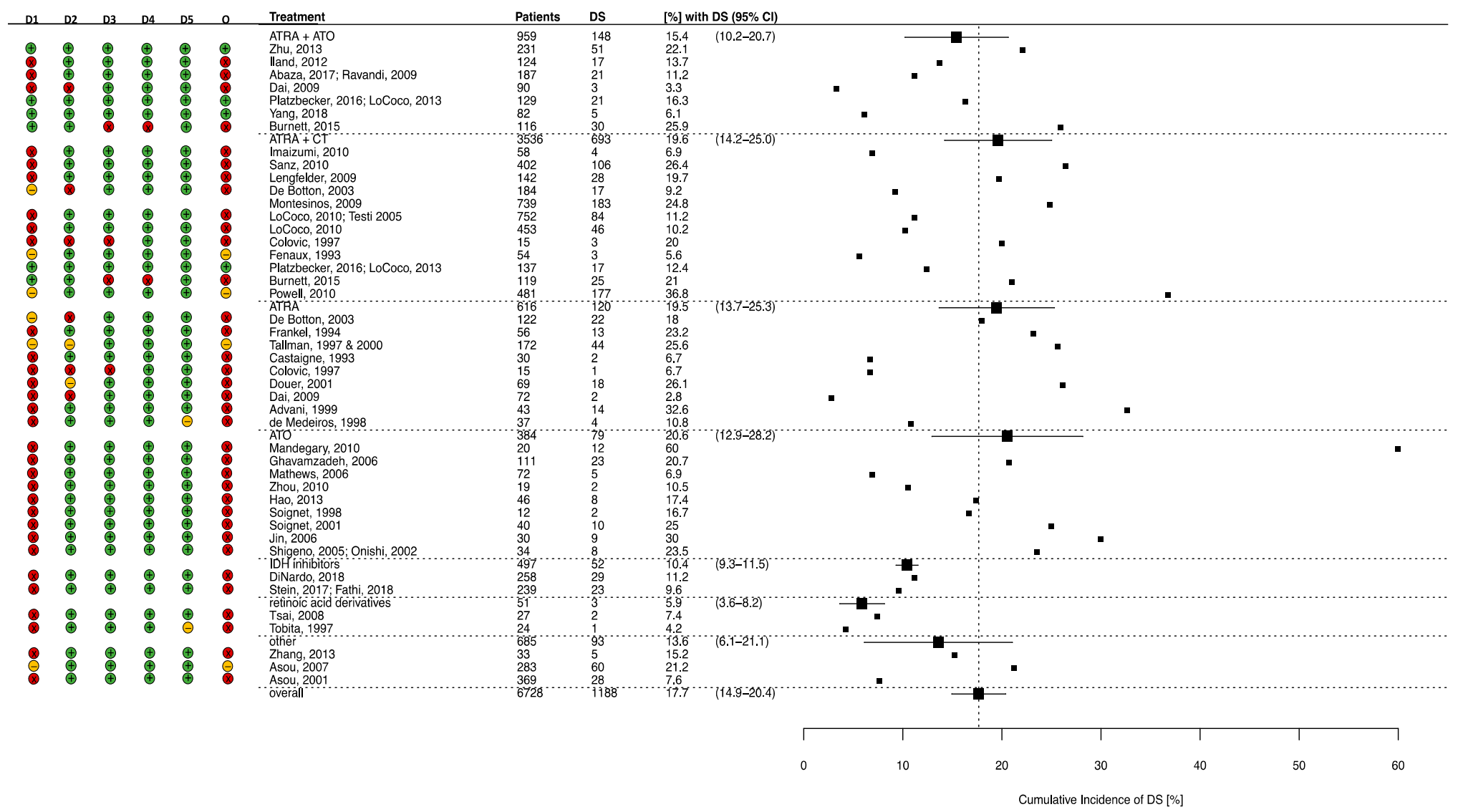

Figure 2. Cumulative incidence of Differentiation Syndrome in the clinical trials grouped by treatment regimen arm. Abbreviations: D = Dimension, D1-D5: Dimensions derived from Risk of Bias Tool (D1: bias arising from the randomization process; D2: Bias due to deviations from intended interventions; D3: Bias due to missing outcome data; D4: Bias in the measurement of outcome; D5: Bias in the selection of cases). O: Overall risk of bias assessment. DS: differentiation syndrome, CI: confidence interval, ATRA: all-trans retinoic acid, ATO: arsenic trioxide, CT: chemotherapy, IDH: isocitrate dehydrogenase. Notes: Grouped by treatment arm, not individual trials. Green indicates a low risk of bias; Yellow indicates some concerns; Red indicates a high risk of bias. The risk of bias was generated using the risk of bias visualization tool (https://www.riskofbias.info/welcome/robvis-visualization-tool). 
Bias arising from the randomization process

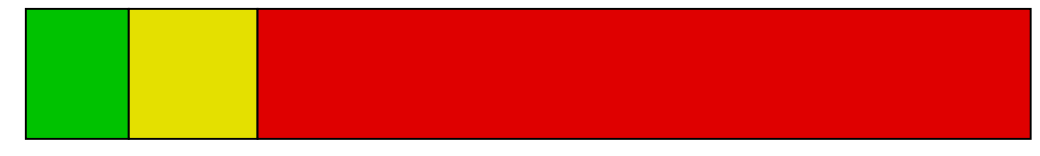

Bias due to deviations from intended interventions

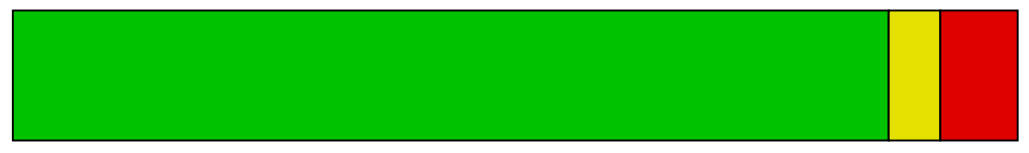

Bias due to missing outcome data

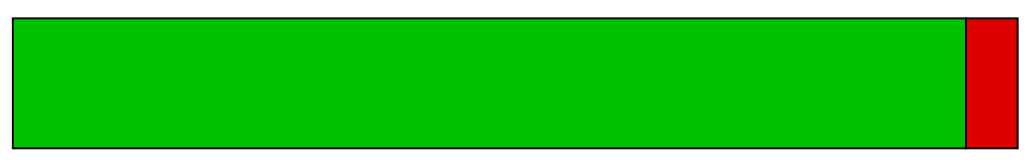

Bias in measurement of the outcome

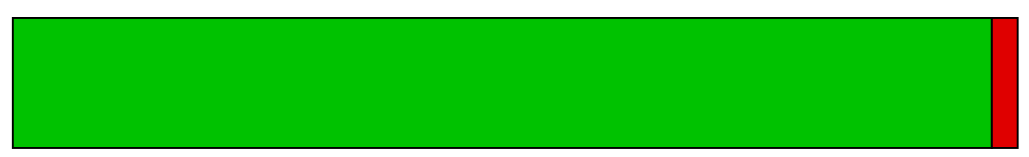

Bias in selection of the reported result

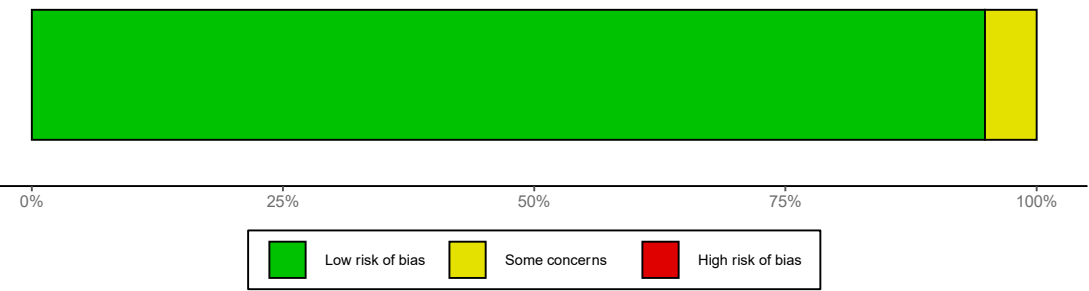

Figure 3. Risk of bias summary for all included studies $(n=39)$. Note: Figure generated using the Risk of Bias visualization tool available at: https: //www.riskofbias.info/welcome/robvis-visualization-tool. 


\section{Discussion}

In this review, we examined the incidence of DS with all differentiating agents, enabling an assessment of the incidence with the IDH-inhibitors compared to older existing therapies traditionally used in APL treatment. Overall, the cumulative incidence of DS in all therapies was $17.7 \%$. DS's incidence was notably lower in trials with IDH-inhibitors (10.4\%) and retinoic acid derivatives $(5.9 \%)$ than regimens based on the old differentiating agents-namely ATRA and ATO. The overall mortality rate among those with DS was relatively low, with no deaths reported among the IDH-inhibitors. The reported median time to onset was substantially longer among the IDH-inhibitor enasidenib (48 days) than the other existing therapies (11 days) (Supplementary Table S1).

In our review, we identified that most studies and treatment arms were for the combination use of ATRA and chemotherapy. This was an expected finding, as it was the recommended first-line treatment regimen for all APL patients before the approval of ATO in combination with ATRA for the first-line treatment of low- and intermediate-risk APL in 2018 [2,96]. Dividing the patients into their respective treatment groups, we observed no difference in DS incidence between the groups treated with regimens based on the old differentiating agents (ATRA and ATO), thereby indicating that no one of the ATRA- and ATO-based treatment regimens seem to pose an increased risk for DS development compared to each other. We also did not observe substantial differences in the proportion of deaths or time to DS onset among the ATRA and ATO treatment regimens.

In the patients treated with the new IDH-inhibitors ivosidenib and enasidenib and the patients treated with the retinoic acid derivatives, tamibarotene, and bexarotene, the incidence of DS was below the overall cumulative incidence. We note that the IDH-inhibitors, contrary to the ATRA- and ATO-based regimens, were used to treat non-M3 AML, as was the retinoic acid derivative bexarotene. Therefore, it is possible that non-M3 AML patients are less prone to developing DS during their remission induction. What might further contribute to a lower DS incidence in IDH-inhibitors and retinoic acid derivatives is that three of the four clinical trials were phase 1 and using dose escalation, meaning that part of the patients received very low doses of the differentiating drugs. Since our estimates of the incidence of DS in the treatment with the IDH-inhibitors, ivosidenib and enasidenib, is based on the published phase 1/2 clinical trials, we may be underestimating the real-world incidence and mortality of DS associated with IDH-inhibitors [13,14,91].

Additionally, the delayed time to onset observed in the IDH-inhibitors indicates that either DS develops later in these patients, or there was a delay in clinical detection. A recent systematic analysis by the US FDA conducted an algorithmic analysis to improve the capture of DS cases in patients using IDH-inhibitors as many of the signs and symptoms of DS may not be initially recognized [14]. The authors concluded that DS was likely common with the IDH-inhibitors, and increased awareness of the early signs and symptoms is needed to decrease the potential for severe or fatal complications. Lastly, due to the lack of diagnostic markers, the diagnosis of DS in ATRA and ATO treatment relies on several unspecific symptoms. Whether these diagnostic criteria can be applied to detect DS in non-M3 AML patients reliably, or whether the application of the same criteria might lead to further underdiagnosis, is unclear [1]. Moreover, we note that the complete remission rates were substantially higher in the ATRA/ATO patients than those treated with IDH-inhibitors. Thus, without clinical awareness and standardized diagnostic criteria of DS with IDH-inhibitors, it is possible that the symptoms of DS are incorrectly categorized as disease progression and subsequently under-recorded. Thus, without clinical awareness and standardized diagnostic criteria of DS with IDH-inhibitors, it is possible that the symptoms of DS are incorrectly categorized as disease progression and subsequently under-recorded. Given this, we believe the FDA boxed warning $[18,19]$ is an appropriate measure to alert health professionals to this potentially fatal ADR to ensure time and appropriate treatment $[18,19]$. We also note that the market authorization application submitted to the EMA for enasidenib was withdrawn due to a limited benefit/risk ratio [20]. Thus, it is evident that large clinical trials, pharmacovigilance, and post-market observational studies of ivosidenib and enasidenib are necessary to characterize the risk of DS. 
Our systematic literature review identified that the first reporting of DS in non-M3 AML was in 2008 in patients treated with bexarotene [93,97], an approved therapy for treating cutaneous T-cell lymphomas resistant to at least one prior systemic therapy [98]. In 2008, Tsai et al. reported on a phase 1 study of bexarotene conducted in patients with non-M3 AML, where two of the 27 patients in the trial experienced DS [93]. We did not identify any literature reports of bexarotene provoking DS in its approved indication. From the preclinical studies, the mechanism of action of both enasidenib and ivosidenib induces differentiation in cancer cells providing pathophysiologic plausibility to the observed occurrence of DS outside of APL $[13,91]$. Moreover, we saw that DS was associated with two additional drugs trialed to treat APL, realgar-indigo naturalis formulation (RIF), and tamibarotene. In two of the ATRA trials, we identified two studies, including RIF, a traditional Chinese medicine with the main active ingredients tetraarsenic tetrasulfide $\left(\mathrm{As}_{4} \mathrm{~S}_{4}\right)$, indirubin, and tanshinone IIA [40,53]. In both studies from China, RIF was an oral replacement for ATO [99]. Tamibarotene is a derivative of retinoic acid and approved in Japan to treat relapsed and refractory APL [100]. Currently, a phase 2 trial of tamibarotene for the treatment of AML and myelodysplastic syndrome is ongoing in the USA (NCT02807558) [101] and was included in our review as a single-agent drug in a phase 1 study as a second-line treatment [88]. A multicenter phase 2 study on tamibarotene in adults with relapsed or refractory APL after treatment with ATRA and ATO (NCT00520208) found an overall response rate of $64 \%$. However, the median overall survival was 9.5 months, and 7 of 9 patients relapsed after a median of 4.6 months [102].

Due to the inclusion of many non-randomized clinical trials, most of the clinical trials in this systematic review had a high risk of bias. However, we believe the high risk of bias noted does not necessarily disqualify these studies or their results but rather presents a limitation to the assessment tool. However, we note only marginal changes in the weighted mean incidence when including only those trials with a low risk of bias [40,51-53] or some concerns [31,34,43,68,82]. The most considerable change was among patients treated with ATRA+CT (19.6\% vs. $29.3 \%)$, which is likely due to the inclusion of only three studies with DS incidence ranging from $5.6 \%$ to $36.8 \%$.

Our systematic review has notable limitations. We performed a single-engine search in PubMed, which primarily indexes medical literature and comprises more than 30 million citations [103]. As such, we may have missed some articles that are not PubMed indexed. However, within PubMed, we chose inclusive search terms with no exclusion based on disease type. Additionally, we conducted a thorough citation search of all relevant articles, which identified 25 additional articles to minimize this risk. As we note that this may limit the reproducibility of the results, we have provided the citations of the articles identified by hand search to improve transparency. Given the small number of studies with appropriate randomization, the clinical trials were subject to a high risk of bias. Although we did not identify substantial differences between the findings of those trials with a low or moderate risk of bias, as compared to the high risk of bias, we believe future analyses of large randomized trials are needed, particularly for the new IDH-inhibitors.

Moreover, while the outcome of DS incidence did not change when assessing those with a low or moderate risk of bias, we expect that clinical trial reports are subject to misclassification bias, rooted in the lack of universally applied diagnostic criteria for DS and the unspecific nature of its symptoms, particularly for the non-M3 AML patients treated with IDH-inhibitors [7,104]. Another limitation is that we focused our review on the occurrence of DS and, therefore, did not summarize information on disease-free survival or relapse rate. We note that there is no demonstrated benefit of ATRA combination with conventional chemotherapy, and consolidation in randomized studies is needed. However, clinical trials have shown a statistically significant reduction in relapse rates and longer disease-free survival $[30,70]$.

Finally, while we acknowledge the limitation of comparing treatment regimens for M3 and non-M3 acute leukemia, we believe a review comparing different treatment regimens causing DS improves the understanding of this potentially fatal adverse drug reaction and highlights the significance in the newly developed IDH-inhibitors. Therefore, we hope to raise awareness of DS among treating 
clinicians and encourage researchers to conduct observational studies to provide real-world clinical evidence to further elucidate the development of DS in both M3 and non-M3 AML. Moreover, future research undertaking clinical development of potential differentiating agents should consider and assess the potential for DS. We believe this is necessary considering that differentiating therapy is a promising and evolving therapeutic field [17], yet the unspecific nature of DS symptoms makes it difficult to diagnose if occurring unexpectedly in non-traditional patient groups.

\section{Conclusions}

This research aimed to contextualize the unexpected occurrence of DS in the clinical trials, with a particular focus on the comparison of IDH-inhibitors (ivosidenib and enasidenib) to existing ATRA and ATO therapies. While we found no difference between the ATRA- and ATO-based regimens used in APL treatment, DS occurred less frequently during treatment with IDH-inhibitors in non-M3 AML. However, this may be due to underreporting and lack of existing diagnostic criteria in this patient group. In light of the unexpected occurrence of DS in non-M3 AML, and considering that differentiation therapy is a promising research area expected to contribute to future cancer treatments, we consider it crucial to raise awareness of DS among treating clinicians and researchers to close this knowledge gap. Due to the paucity of data and rarity of DS in IDH-inhibitors, we believe the warnings from competent authorities are justified, and treating oncologists should be mindful of the potential of DS in non-M3 AML patients. However, further large randomized trials or studies using real-world data are required to provide further insights.

Supplementary Materials: The following are available online at http://www.mdpi.com/2077-0383/9/10/3342/s1, Table S1: Complete data extraction of included clinical trials $(n=39)$ and patient characteristics, treatment regimens, and outcomes.

Author Contributions: Conceptualization, S.W. and A.M.B.; methodology, L.G. and A.M.B.; software, A.M.B.; literature review, L.G. and L.H.; validation, L.G. and L.H.; formal analysis, L.G.; writing-original draft preparation, L.G.; writing-review and editing, L.G., S.W., L.H., and A.M.B.; visualization, L.G.; supervision, S.W. and A.M.B.; project administration, A.M.B.; funding acquisition, n/a. All authors meet the authorship criteria and have read and agreed to the published version of the manuscript.

Funding: This research received no external funding.

Conflicts of Interest: S.W. is a member of the Pharmacovigilance Risk Assessment Committee (PRAC) of the EMA and the head of research at Tox Info Suisse. The views expressed in this article are the authors' personal views and may not be understood or quoted as being made on behalf of or reflecting the position of the EMA or one of its committees or working parties. All other authors (L.G., L.H., A.M.B.) declare no conflicts of interest related to this work.

\section{References}

1. Montesinos, P.; Bergua, J.M.; Vellenga, E.; Rayon, C.; Parody, R.; de la Serna, J.; Leon, A.; Esteve, J.; Milone, G.; Deben, G.; et al. Differentiation syndrome in patients with acute promyelocytic leukemia treated with all-trans retinoic acid and anthracycline chemotherapy: Characteristics, outcome, and prognostic factors. Blood 2009, 113, 775-783. [CrossRef] [PubMed]

2. Available online: https://www.uptodate.com/contents/initial-treatment-of-acute-promyelocytic-leukemia-inadults? search=acute (accessed on 9 August 2019).

3. Leukemia \& Lymphoma Society. Acute Promyelocytic Leukemia Facts. Available online: https: //www.lls.org/sites/default/files/National/USA/Pdf/Publications/APL_FactSheet_10_15FINAL.pdf (accessed on 9 August 2019).

4. Ryan, M.M. Acute Promyelocytic Leukemia: A Summary. J. Adv. Pr. Oncol. 2018, 9, 178-187.

5. Lallemand-Breitenbach, V.; De Thé, H. Retinoic acid plus arsenic trioxide, the ultimate panacea for acute promyelocytic leukemia? Blood 2013, 122, 2008-2010. [CrossRef] [PubMed]

6. Jillella, A.; Kota, V. The global problem of early deaths in acute promyelocytic leukemia: A strategy to decrease induction mortality in the most curable leukemia. Blood Rev. 2018, 32, 89-95. [CrossRef]

7. Available online: https://www.uptodate.com/contents/differentiation-syndrome-associated-with-treatmentof-acute-leukemia (accessed on 15 August 2019). 
8. Montesinos, P.; Sanz, M.A. The differentiation syndrome in patients with acute promyelocytic leukemia: experience of the Pethema group and review of the literature. Mediterr. J. Hematol. Infect. Dis. 2011, 3, 2011059. [CrossRef]

9. Frankel, S.R.; Eardley, A.; Lauwers, G.; Weiss, M.; Warrell, R.P. The "Retinoic Acid Syndrome" in Acute Promyelocytic Leukemia. Ann. Intern. Med. 1992, 117, 292-296. [CrossRef]

10. Sanz, M.A.; Montesinos, P. How we prevent and treat differentiation syndrome in patients with acute promyelocytic leukemia. Blood 2014, 123, 2777-2782. [CrossRef]

11. Available online: https://www.meddra.org/ (accessed on 15 August 2019).

12. Fathi, A.T.; Dinardo, C.D.; Kline, I.; Kenvin, L.; Gupta, I.; Attar, E.C.; Stein, E.M.; De Botton, S.; for the AG221-C-001 Study Investigators. Differentiation Syndrome Associated With Enasidenib, a Selective Inhibitor of Mutant Isocitrate Dehydrogenase 2. JAMA Oncol. 2018, 4, 1106-1110. [CrossRef]

13. Dinardo, C.D.; Stein, E.M.; De Botton, S.; Roboz, G.J.; Altman, J.K.; Mims, A.S.; Swords, R.; Collins, R.H.; Mannis, G.N.; Pollyea, D.A.; et al. Durable Remissions with Ivosidenib inIDH1-Mutated Relapsed or Refractory AML. N. Engl. J. Med. 2018, 378, 2386-2398. [CrossRef]

14. Norsworthy, K.; Mulkey, F.; Scott, E.C.; Ward, A.F.; Przepiorka, D.; Charlab, R.; Dorff, S.E.; Deisseroth, A.; Kazandjian, D.; Sridhara, R.; et al. Differentiation syndrome with ivosidenib and enasidenib treatment in patients with relapsed or refractory IDH-mutated AML: A U.S. Food and Drug Administration systematic analysis. Clin. Cancer Res. 2020, 26. [CrossRef]

15. Zeidner, J.F. Differentiating the Differentiation Syndrome Associated with IDH Inhibitors in AML. Clin. Cancer Res. 2020, 26. [CrossRef] [PubMed]

16. Food and Drug Administration. FDA approves ivosidenib as first-line treatment for AML with IDH1 mutation. Available online: https://www.fda.gov/drugs/resources-information-approved-drugs/ fda-approves-ivosidenib-first-line-treatment-aml-idh1-mutation (accessed on 15 August 2019).

17. De Thé, H. Differentiation therapy revisited. Nat. Rev. Cancer 2017, 18, 117-127. [CrossRef]

18. IDHIFA—Full Prescribing Information. Available online: https://packageinserts.bms.com/pi/pi_idhifa.pdf (accessed on 15 October 2020).

19. Available online: https://www.tibsovopro.com/pdf/prescribinginformation.pdf (accessed on 15 November 2019).

20. Available online: https://www.ema.europa.eu/en/documents/medicine-qa/questions-answers-withdrawalapplication-marketing-authorisation-idhifa-enasidenib_en.pdf (accessed on 15 November 2019).

21. Schüklenk, U. Retraction watch. Bioethics 2012, 26. [CrossRef] [PubMed]

22. Sterne, J.A.C.; Savović, J.; Page, M.J.; Elbers, R.G.; Blencowe, N.S.; Boutron, I.; Cates, C.J.; Cheng, H.Y.; Corbett, M.S.; Eldridge, S.M.; et al. RoB 2: A revised tool for assessing risk of bias in randomised trials. BMJ 2019, 28. [CrossRef] [PubMed]

23. Sanz, M.A.; Martín, G.; González, M.; León, A.; Rayón, C.; Rivas, C.; Colomer, D.; Amutio, E.; Capote, F.J.; Milone, G.A.; et al. Risk-adapted treatment of acute promyelocytic leukemia with all-trans-retinoic acid and anthracycline monochemotherapy: A multicenter study by the PETHEMA group. Blood 2003, 103, 1237-1243. [CrossRef] [PubMed]

24. Ohno, R.; Yoshida, H.; Fukutani, H.; Naoe, T.; Ohshima, T.; Kyo, T.; Endoh, N.; Fujimoto, T.; Kobayashi, T.; Hiraoka, A. Multi-institutional study of all-trans-retinoic acid as a differentiation therapy of refractory acute promyelocytic leukemia. Leukaemia Study Group of the Ministry of Health and Welfare. Leukemia 1993, 7, 1722-1727. [PubMed]

25. Mourad, Y.A.; Jabr, F.; Salem, Z. Scrotal ulceration induced by all-trans retinoic acid in a patient with acute promyelocytic leukemia. Int. J. Dermatol. 2005, 44, 67-68. [CrossRef]

26. Tallman, M.S.; Nabhan, C.; Feusner, J.H.; Rowe, J.M. Acute promyelocytic leukemia: Evolving therapeutic strategies. Blood 2002, 99, 759-767. [CrossRef]

27. Zhou, J.; Zhang, Y.; Li, J.; Li, X.; Hou, J.; Zhao, Y.; Liu, X.; Han, X.; Hu, L.; Wang, S.; et al. Single-agent arsenic trioxide in the treatment of children with newly diagnosed acute promyelocytic leukemia. Blood 2010, 115, 1697-1702. [CrossRef]

28. Li, E.-Q.; Xu, L.; Zhang, Z.-Q.; Xiao, Y.; Guo, H.-X.; Luo, X.-Q.; Hu, Q.; Lai, D.-B.; Tu, L.-M.; Jin, R.-M. Retrospective analysis of 119 cases of pediatric acute promyelocytic leukemia: Comparisons of four treatment regimes. Exp. Ther. Med. 2012, 4, 93-98. [CrossRef] 
29. Soignet, S.L.; Frankel, S.R.; Douer, D.; Tallman, M.S.; Kantarjian, H.; Calleja, E.; Stone, R.M.; Kalaycio, M.; Scheinberg, D.A.; Steinherz, P.; et al. United States Multicenter Study of Arsenic Trioxide in Relapsed Acute Promyelocytic Leukemia. J. Clin. Oncol. 2001, 19, 3852-3860. [CrossRef] [PubMed]

30. Sanz, M.A.; Martín, G.; Rayón, C.; Esteve, J.; González, M.; Díaz-Mediavilla, J.; Bolufer, P.; Barragán, E.; Terol, M.J.; González, J.D.; et al. A modified AIDA protocol with anthracycline-based consolidation results in high antileukemic efficacy and reduced toxicity in newly diagnosed PML/RARalpha-positive acute promyelocytic leukemia. PETHEMA group. Blood 1999, 94, 3015-3021. [PubMed]

31. Powell, B.L.; Moser, B.; Stock, W.; Gallagher, R.E.; Willman, C.L.; Stone, R.M.; Rowe, J.M.; Coutre, S.; Feusner, J.H.; Gregory, J.; et al. Arsenic trioxide improves event-free and overall survival for adults with acute promyelocytic leukemia: North American Leukemia Intergroup Study C9710. Blood 2010, 116, 3751-3757. [CrossRef]

32. Fenaux, P.; Chastang, C.; Chevret, S.; Sanz, M.; Dombret, H.; Archimbaud, E.; Fey, M.; Rayon, C.; Huguet, F.; Sotto, J.J.; et al. A randomized comparison of all transretinoic acid (ATRA) followed by chemotherapy and ATRA plus chemotherapy and the role of maintenance therapy in newly diagnosed acute promyelocytic leukemia. The European APL Group. Blood 1999, 94, 1192-1200. [CrossRef] [PubMed]

33. Gaínza, F.J.; García, J.; Iruretagoyena, J.R. Continuous hemofiltration in the management of 'retinoic acid syndrome'. Leuk. Res. 1997, 21, 891. [CrossRef]

34. Asou, N.; Kishimoto, Y.; Kiyoi, H.; Okada, M.; Kawai, Y.; Tsuzuki, M.; Horikawa, K.; Matsuda, M.; Shinagawa, K.; Kobayashi, T.; et al. A randomized study with or without intensified maintenance chemotherapy in patients with acute promyelocytic leukemia who have become negative for PML-RAR $\alpha$ transcript after consolidation therapy: The Japan Adult Leukemia Study Group (JALSG) APL97 study. Blood 2007, 110, 59-66. [CrossRef]

35. Burnett, A.K.; Grimwade, D.; Solomon, E.; Wheatley, K.; Goldstone, A.H. Presenting white blood cell count and kinetics of molecular remission predict prognosis in acute promyelocytic leukemia treated with all-trans retinoic acid: Result of the Randomized MRC Trial. Blood 1999, 93, 4131-4143. [CrossRef]

36. Mahendra, P.; Harman, K.; Phillips, M.; Gunning, K.; Marcus, R. Rapid progression of'retinoic acid syndrome'in the hypogranular variant of acute promyelocytic leukaemia, despite treatment with dexamethasone and conventional chemotherapy. Int. J. Lab. Hematol. 2008, 16, 371-374. [CrossRef]

37. Metage, C.; Hazarika, B.; Sarma, J.; Karwa, R. Retinoic acid syndrome in a elderly male with psoriasis- A case report. Respir. Med. Case Rep. 2018, 24, 81-83. [CrossRef]

38. Hu, J.; Shen, Z.-X.; Sun, G.-L.; Chen, S.-J.; Wang, Z.; Chen, Z. Long-term survival and prognostig study in acute promyelocytic leukemia treated with all-trans retinoic acid, chemotherapy, and As2O3-An experience of 120 patients at a single institution. Int. J. Hematol. 1999, 70, 248-260.

39. Avvisati, G.; Coco, F.L.; Diverio, D.; Falda, M.; Ferrara, F.; Lazzarino, M.; Russo, D.; Petti, M.; Mandelli, F. AIDA (all-trans retinoic acid + idarubicin) in newly diagnosed acute promyelocytic leukemia: A Gruppo Italiano Malattie Ematologiche Maligne dell'Adulto (GIMEMA) pilot study. Blood 1996, 88, 1390-1398. [CrossRef]

40. Yang, M.-H.; Wan, W.-Q.; Luo, J.-S.; Zheng, M.-C.; Huang, K.; Yang, L.-H.; Mai, H.-R.; Li, J.; Chen, H.-Q.; Sun, X.-F.; et al. Multicenter randomized trial of arsenic trioxide and Realgar-Indigo naturalisformula in pediatric patients with acute promyelocytic leukemia: Interim results of the SCCLG-APL clinical study. Am. J. Hematol. 2018, 93, 1467-1473. [CrossRef] [PubMed]

41. Charles, K.S.; Kanaa, M.; Winfield, D.; Reilly, J.T. Scrotal ulceration during all-trans retinoic (ATRA) therapy for acute promyelocytic leukaemia. Int. J. Lab. Hematol. 2000, 22, 171-174. [CrossRef] [PubMed]

42. Sakakura, M.; Nishii, K.; Usui, E.; Monma, F.; Tsukada, T.; Shikua, H. Bilateral Osteonecrosis of the Head of the Femur during Treatment with Retinoic Acid in a Young Patient with Acute Promyelocytic Leukemia. Int. J. Hematol. 2006, 83, 252-253. [CrossRef] [PubMed]

43. Fenaux, P.; Le Deley, M.C.; Castaigne, S.; Archimbaud, E.; Chomienne, C.; Link, H.; Guerci, A.; Duarte, M.; Daniel, M.T.; Bowen, D.; et al. Effect of all transretinoic acid in newly diagnosed acute promyelocytic leukemia. Results of a multicenter randomized trial. Blood 1993, 82, 3241-3249. [CrossRef]

44. Sanz, M.A.; Montesinos, P.; Rayón, C.; Holowiecka, A.; De La Serna, J.; Milone, G.; De Lisa, E.; Brunet, S.; Rubio, V.; Ribera, J.; et al. Risk-adapted treatment of acute promyelocytic leukemia based on all-trans retinoic acid and anthracycline with addition of cytarabine in consolidation therapy for high-risk patients: Further improvements in treatment outcome. Blood 2010, 115, 5137-5146. [CrossRef] 
45. Iland, H.J.; Bradstock, K.; Seymour, J.; Hertzberg, M.; Grigg, A.; Taylor, K.; Catalano, J.; Cannell, P.; Horvath, N.; Deveridge, S.; et al. Results of the APML3 trial incorporating all-trans-retinoic acid and idarubicin in both induction and consolidation as initial therapy for patients with acute promyelocytic leukemia. Haematologica 2011, 97, 227-234. [CrossRef]

46. Raanani, P.; Segal, E.; Levi, I.; Bercowicz, M.; Berkenstat, H.; Avigdor, A.; Perel, A.; Ben-Bassat, I. Diffuse Alveolar Hemorrhage in Acute Promyelocytic Leukemia Patients Treated with ATRA-A Manifestation of the Basic Disease or the Treatment. Leuk. Lymphoma 2000, 37, 605-610. [CrossRef]

47. Goldschmidt, N.; Gural, A.; Ben Yehuda, D. Extensive Splenic Infarction, Deep Vein Thrombosis and Pulmonary Emboli Complicating Induction Therapy with All-trans-retinoic Acid (ATRA) for Acute Promyelocytic Leukemia. Leuk. Lymphoma 2003, 44, 1433-1437. [CrossRef]

48. Burnett, A.K.; Russell, N.H.; Hills, R.K.; Bowen, D.T.; Kell, J.; Knapper, S.; Morgan, Y.G.; Lok, J.; Grech, A.; Jones, G.; et al. Arsenic trioxide and all-trans retinoic acid treatment for acute promyelocytic leukaemia in all risk groups (AML17): Results of a randomised, controlled, phase 3 trial. Lancet Oncol. 2015, 16, 1295-1305. [CrossRef]

49. Abaza, Y.; Kantarjian, H.; Garcia-Manero, G.; Estey, E.; Borthakur, G.; Jabbour, E.; Faderl, S.; O’Brien, S.; Wierda, W.; Pierce, S.; et al. Long-term outcome of acute promyelocytic leukemia treated with all-trans-retinoic acid, arsenic trioxide, and gemtuzumab. Blood 2017, 129, 1275-1283. [CrossRef]

50. Mandelli, F.; Diverio, D.; Avvisati, G.; Luciano, A.; Barbui, T.; Bernasconi, C.; Broccia, G.; Cerri, R.; Falda, M.; Fioritoni, G.; et al. Molecular Remission in PML/RARA-Positive Acute Promyelocytic Leukemia by Combined All-Trans Retinoic Acid and Idarubicin (AIDA) Therapy. Blood 1997, 90, 1014-1021. [PubMed]

51. Platzbecker, U.; Avvisati, G.; Cicconi, L.; Thiede, C.; Paoloni, F.; Vignetti, M.; Ferrara, F.; Divona, M.; Albano, F.; Efficace, F.; et al. Improved Outcomes With Retinoic Acid and Arsenic Trioxide Compared With Retinoic Acid and Chemotherapy in Non-High-Risk Acute Promyelocytic Leukemia: Final Results of the Randomized Italian-German APL0406 Trial. J. Clin. Oncol. 2017, 35, 605-612. [CrossRef]

52. Lo-Coco, F.; Avvisati, G.; Vignetti, M.; Thiede, C.; Orlando, S.M.; Iacobelli, S.; Ferrara, F.; Fazi, P.; Cicconi, L.; Di Bona, E.; et al. Retinoic Acid and Arsenic Trioxide for Acute Promyelocytic Leukemia. N. Engl. J. Med. 2013, 369, 111-121. [CrossRef] [PubMed]

53. Zhu, H.-H.; Wu, D.-P.; Jin, J.; Li, J.-Y.; Ma, J.; Wang, J.-X.; Jiang, H.; Chen, S.-J.; Huang, X.-J. Oral Tetra-Arsenic Tetra-Sulfide Formula Versus Intravenous Arsenic Trioxide As First-Line Treatment of Acute Promyelocytic Leukemia: A Multicenter Randomized Controlled Trial. J. Clin. Oncol. 2013, 31, 4215-4221. [CrossRef] [PubMed]

54. Dai, C.-W.; Zhang, G.-S.; Shen, J.-K.; Zheng, W.-L.; Pei, M.-F.; Xu, Y.-X.; Cao, Y.-X.; Yi, Y.; Yang, J.-J.; Peng, H.-L.; et al. Use of All-trans Retinoic Acid in Combination with Arsenic Trioxide for Remission Induction in Patients with Newly Diagnosed Acute Promyelocytic Leukemia and for Consolidation/Maintenance in CR Patients. Acta Haematol. 2009, 121, 1-8. [CrossRef] [PubMed]

55. Ravandi, F.; Estey, E.; Jones, D.; Faderl, S.; O’Brien, S.; Fiorentino, J.; Pierce, S.; Blamble, D.; Estrov, Z.; Wierda, W.; et al. Effective Treatment of Acute Promyelocytic Leukemia With All-Trans-Retinoic Acid, Arsenic Trioxide, and Gemtuzumab Ozogamicin. J. Clin. Oncol. 2009, 27, 504-510. [CrossRef]

56. Testi, A.M.; Biondi, A.; Albano, F.; Moleti, M.L.; Giona, F.; Vignetti, M.; Menna, G.; Locatelli, F.; Pession, A.; Barisone, E.; et al. GIMEMA-AIEOPAIDA protocol for the treatment of newly diagnosed acute promyelocytic leukemia (APL) in children. Blood 2005, 106, 447-453. [CrossRef]

57. De Botton, S.; for the European Apl Group; Chevret, S.; Coiteux, V.; Dombret, H.; Sanz, M.A.; San-Miguel, J.F.; Caillot, D.; Vekhoff, A.; Gardembas, M.; et al. Early onset of chemotherapy can reduce the incidence of ATRA syndrome in newly diagnosed acute promyelocytic leukemia (APL) with low white blood cell counts: results from APL 93 trial. Leukemia 2003, 17, 339-342. [CrossRef]

58. Battistella, M.; Burry, L.; Seki, J.T. Retinoic acid syndrome after one dose of all-transretinoic acid. J. Oncol. Pharm. Pr. 2004, 10, 149-154. [CrossRef]

59. Kienast, J.; Stelljes, M.; Berning, B.; Kröger, M.; Sauerland, M.C.; Heinecke, A.; Schoch, C.; Wörmann, B.; Büchner, T.; Hiddemann, W.; et al. Satelite Symposium V, Meet-the-Professor Sessions I and II, Main Sessions I-IX. Ann. Hematol. 2004, 83, 59-137. [CrossRef]

60. Levasseur, S.D.; Tantiworawik, A.; Maberley, D.A.L. All-Trans Retinoic Acid Differentiation Syndrome Chorioretinopathy. Retin. Cases Brief Rep. 2013, 7, 46-49. [CrossRef] 
61. Asou, N.; Adachi, K.; Tamura, J.; Kanamaru, A.; Kageyama, S.; Hiraoka, A.; Omoto, E.; Sakamaki, H.; Tsubaki, K.; Saito, K.; et al. All-trans retinoic acid therapy for newly diagnosed acute promyelocytic leukemia: comparison with intensive chemotherapy. The Japan Adult Leukemia Study Group (JALSG). Cancer Chemother. Pharmacol. 1997, 40, 30-35. [CrossRef]

62. Newman, A.; Leung, B.; Richards, A.; Campbell, T.; Wellwood, J.; Imrie, F. Two cases of differentiation syndrome with ocular manifestations in patients with acute promyelocytic leukaemia treated with all-trans retinoic acid and arsenic trioxide. Am. J. Ophthalmol. Case Rep. 2018, 9, 106-111. [CrossRef] [PubMed]

63. De Botton, S.; Coiteux, V.; Chevret, S.; Rayon, C.; Vilmer, E.; Sanz, M.A.; De La Serna, J.; Philippe, N.; Baruchel, A.; Leverger, G.; et al. Outcome of Childhood Acute Promyelocytic Leukemia With All-Trans-Retinoic Acid and Chemotherapy. J. Clin. Oncol. 2004, 22, 1404-1412. [CrossRef]

64. Mathews, V.; George, B.; Lakshmi, K.M.; Viswabandya, A.; Bajel, A.; Balasubramanian, P.; Shaji, R.V.; Srivastava, V.M.; Srivastava, A.; Chandy, M. Single-agent arsenic trioxide in the treatment of newly diagnosed acute promyelocytic leukemia: Durable remissions with minimal toxicity. Blood 2006, 107, 2627-2632. [CrossRef] [PubMed]

65. Kakkar, N.; Dhameja, N.; Jasmina; Das, A.; Radotra, B.; Varma, S. ATRA syndrome with extensive organ infiltration. Am. J. Hematol. 2002, 71, 62-64. [CrossRef]

66. Chou, W.-C.; Tang, J.-L.; Yao, M.; Liang, Y.J.; Lee, F.Y.; Lin, M.T.; Wang, C.H.; Shen, M.C.; Chen, Y.C.; Tien, H.-F. Clinical and biological characteristics of acute promyelocytic leukemia in Taiwan: a high relapse rate in patients with high initial and peak white blood cell counts during all-trans retinoic acid treatment. Leukemia 1997, 11, 921-928. [CrossRef]

67. Yang, C.-L.; Shen, K.; Huang, J. Diffuse Pulmonary Alveolar Hemorrhage Secondary to All-Trans-Retinoic Acid in Acute Promyelocytic Leukemia. Chin. Med J. 2018, 131, 2386-2387. [CrossRef]

68. Tallman, M.S.; Andersen, J.W.; Schiffer, C.A.; Appelbaum, F.R.; Feusner, J.H.; Ogden, A.; Shepherd, L.; Willman, C.; Bloomfield, C.D.; Rowe, J.M.; et al. All-trans-Retinoic Acid in Acute Promyelocytic Leukemia. N. Engl. J. Med. 1997, 337, 1021-1028. [CrossRef]

69. Estey, E.; Garcia-Manero, G.; Ferrajoli, A.; Faderl, S.; Verstovsek, S.; Jones, D.; Kantarjian, H. Use of all-trans retinoic acid plus arsenic trioxide as an alternative to chemotherapy in untreated acute promyelocytic leukemia. Blood 2006, 107, 3469-3473. [CrossRef]

70. Lo-Coco, F.; Avvisati, G.; Vignetti, M.; Breccia, M.; Gallo, E.; Rambaldi, A.; Paoloni, F.; Fioritoni, G.; Ferrara, F.; Specchia, G.; et al. Front-line treatment of acute promyelocytic leukemia with AIDA induction followed by risk-adapted consolidation for adults younger than 61 years: results of the AIDA-2000 trial of the GIMEMA Group. Blood 2010, 116, 3171-3179. [CrossRef]

71. Ades, L.; for the European Apl Group; Chevret, S.; De Botton, S.; Thomas, X.; Dombret, H.; Beve, B.; Sanz, M.A.; Guerci, A.; Miguel, J.S.; et al. Outcome of acute promyelocytic leukemia treated with all trans retinoic acid and chemotherapy in elderly patients: The European group experience. Leukemia 2004, 19, 230-233. [CrossRef] [PubMed]

72. Ghavamzadeh, A.; Alimoghaddam, K.; Ghaffari, S.H.; Rostami, S.; Jahani, M.; Hosseini, R.; Mossavi, A.; Baybordi, E.; Khodabadeh, A.; Iravani, M.; et al. Treatment of acute promyelocytic leukemia with arsenic trioxide without ATRA and/or chemotherapy. Ann. Oncol. 2006, 17, 131-134. [CrossRef]

73. Wiley, J.S.; Firkin, F.C. Reduction of pulmonary toxicity by prednisolone prophylaxis during all-trans retinoic acid treatment of acute promyelocytic leukemia. Australian Leukaemia Study Group. Leukemia 1995, 9, 774-778. [PubMed]

74. Lengfelder, E.; for the German AML Cooperative Group (AMLCG); Haferlach, C.; Saussele, S.; Schultheis, B.; Schnittger, S.; Ludwig, W.-D.; Staib, P.; Aul, C.; Grüeisen, A.; et al. High dose ara-C in the treatment of newly diagnosed acute promyelocytic leukemia: Long-term results of the German AMLCG. Leukemia 2009, 23, 2248-2258. [CrossRef] [PubMed]

75. Asou, N.; Adachi, K.; Tamura, J.-I.; Kanamaru, A.; Kageyama, S.-I.; Hiraoka, A.; Omoto, E.; Akiyama, H.; Tsubaki, K.; Saito, K.; et al. Analysis of prognostic factors in newly diagnosed patients with acute promyelocytic leukemia: the APL92 study of the Japan Adult Leukemia Study Group (JALSG). Cancer Chemother. Pharmacol. 2001, 48, 65-71. [CrossRef]

76. Soignet, S.L.; Maslak, P.; Wang, Z.-G.; Jhanwar, S.; Calleja, E.; Dardashti, L.J.; Corso, D.; DeBlasio, A.; Gabrilove, J.; A Scheinberg, D.; et al. Complete Remission after Treatment of Acute Promyelocytic Leukemia with Arsenic Trioxide. N. Engl. J. Med. 1998, 339, 1341-1348. [CrossRef] 
77. Douer, D.; Estey, E.; Santillana, S.; Bennett, J.M.; Lopez-Bernstein, G.; Boehm, K.; Williams, T. Treatment of newly diagnosed and relapsed acute promyelocytic leukemia with intravenous liposomal all-transretinoic acid. Blood 2001, 97, 73-80. [CrossRef]

78. Ferrara, F.; Finizio, O.; D'Arco, A.; Mastrullo, L.; Cantore, N.; Musto, P. Acute promyelocytic leukemia in patients aged over 60 years: multicenter experience of 34 consecutive unselected patients. Anticancer. Res. 2010, 30, 967-972.

79. De-Medeiros, B.; Strapasson, E.; Pasquini, R.; De-Medeiros, C. Effect of all-trans retinoic acid on newly diagnosed acute promyelocytic leukemia patients: Results of a Brazilian center. Braz. J. Med. Biol. Res. 1998, 31, 1537-1543. [CrossRef]

80. Shigeno, K.; Naito, K.; Sahara, N.; Kobayashi, M.; Nakamura, S.; Fujisawa, S.; Shinjo, K.; Takeshita, A.; Ohno, R.; Ohnishi, K. Arsenic Trioxide Therapy in Relapsed or Refractory Japanese Patients with Acute Promyelocytic Leukemia: Updated Outcomes of the Phase II Study and Postremission Therapies. Int. J. Hematol. 2005, 82, 224-229. [CrossRef]

81. Frankel, S.R.; Eardley, A.; Heller, G.; Berman, E.; Miller, W.H.; Dmitrovsky, E.; Warrell, R.P. All-trans Retinoic Acid for Acute Promyelocytic Leukemia: Results of the New York Study. Ann. Intern. Med. 1994, 120, 278-286. [CrossRef] [PubMed]

82. Tallman, M.S.; Andersen, J.W.; A Schiffer, C.; Appelbaum, F.R.; Feusner, J.H.; Ogden, A.; Shepherd, L.; Rowe, J.M.; François, C.; Larson, R.S.; et al. Clinical description of 44 patients with acute promyelocytic leukemia who developed the retinoic acid syndrome. Blood 2000, 95, 90-95.

83. Ohnishi, K.; Yoshida, H.; Shigeno, K.; Nakamura, S.; Fujisawa, S.; Naito, K.; Shinjo, K.; Fujita, Y.; Matsui, H.; Sahara, N.; et al. Arsenic trioxide therapy for relapsed or refractory Japanese patients with acute promyelocytic leukemia: Need for careful electrocardiogram monitoring. Leukemia 2002, 16, 617-622. [CrossRef]

84. Zhang, Y.; Zhang, Z.; Li, J.; Li, L.; Han, X.; Han, L.; Hu, L.; Wang, S.; Zhao, Y.; Li, X.; et al. Long-term efficacy and safety of arsenic trioxide for first-line treatment of elderly patients with newly diagnosed acute promyelocytic leukemia. Cancer 2012, 119, 115-125. [CrossRef] [PubMed]

85. Camacho, L.H.; Soignet, S.L.; Chanel, S.; Ho, R.; Heller, G.; Scheinberg, D.A.; Ellison, R.; Warrell, R.P. Leukocytosis and the Retinoic Acid Syndrome in Patients With Acute Promyelocytic Leukemia Treated With Arsenic Trioxide. J. Clin. Oncol. 2000, 18, 2620-2625. [CrossRef]

86. Mandegary, A.; Hosseini, R.; Ghaffari, S.H.; Alimoghaddam, K.; Rostami, S.; Ghavamzadeh, A.; Ghahremani, M.H. The expression of p38, ERK1 and Bax proteins has increased during the treatment of newly diagnosed acute promyelocytic leukemia with arsenic trioxide. Ann. Oncol. 2010, 21, 1884-1890. [CrossRef] [PubMed]

87. Iland, H.J.; Bradstock, K.; Supple, S.G.; Catalano, A.; Collins, M.; Hertzberg, M.; Browett, P.; Grigg, A.; Firkin, F.; Hugman, A.; et al. All-trans-retinoic acid, idarubicin, and IV arsenic trioxide as initial therapy in acute promyelocytic leukemia (APML4). Blood 2012, 120, 1570-1580. [CrossRef]

88. Tobita, T.; Takeshita, A.; Kitamura, K.; Ohnishi, K.; Yanagi, M.; Hiraoka, A.; Karasuno, T.; Takeuchi, M.; Miyawaki, S.; Ueda, R.; et al. Treatment With a New Synthetic Retinoid, Am80, of Acute Promyelocytic Leukemia Relapsed From Complete Remission Induced by All-trans Retinoic Acid. Blood 1997, 90, 967-973. [CrossRef]

89. Castaigne, S.; Lefebvre, P.; Chomienne, C.; Suc, E.; Rigal-Huguet, F.; Gardin, C.; Delmer, A.; Archimbaud, E.; Tilly, H.; Janvier, M. Effectiveness and pharmacokinetics of low-dose all-trans retinoic acid $(25 \mathrm{mg} / \mathrm{m} 2)$ in acute promyelocytic leukemia. Blood 1993, 82, 3560-3563. [CrossRef] [PubMed]

90. Čolović, M.D.; Janković, G.M.; Elezović, I.; Vidović, A.; Bila, J.S.; Novak, A.; Babić, D. Effect of all-trans-retinoic acid alone or in combination with chemotherapy in newly diagnosed acute promyelocytic leukaemia. Med. Oncol. 1997, 14, 65-72. [CrossRef] [PubMed]

91. Stein, E.M.; Dinardo, C.D.; Pollyea, D.A.; Fathi, A.T.; Roboz, G.J.; Altman, J.K.; Stone, R.M.; DeAngelo, D.J.; Levine, R.L.; Flinn, I.W.; et al. Enasidenib in mutant IDH2 relapsed or refractory acute myeloid leukemia. Blood 2017, 130, 722-731. [CrossRef] [PubMed]

92. Imaizumi, M.; Tawa, A.; Hanada, R.; Tsuchida, M.; Tabuchi, K.; Kigasawa, H.; Kobayashi, R.; Morimoto, A.; Nakayama, H.; Hamamoto, K.; et al. Prospective study of a therapeutic regimen with all-trans retinoic acid and anthracyclines in combination of cytarabine in children with acute promyelocytic leukaemia: The Japanese childhood acute myeloid leukaemia cooperative study. Br. J. Haematol. 2010, 152, 89-98. [CrossRef] [PubMed] 
93. Tsai, D.E.; Luger, S.M.; Andreadis, C.; Vogl, D.T.; Kemner, A.; Potuzak, M.; Goradia, A.; Loren, A.W.; Perl, A.E.; Schuster, S.J.; et al. A Phase I Study of Bexarotene, a Retinoic X Receptor Agonist, in Non-M3 Acute Myeloid Leukemia. Clin. Cancer Res. 2008, 14, 5619-5625. [CrossRef] [PubMed]

94. Advani, S.H.; Nair, R.; Bapna, A.; Gladstone, B.; Kadam, P.; Saikia, T.K.; Parekh, P.M.; Gopal, R.; Nair, C.N. Acute promyelocytic leukemia: All-trans retinoic acid (ATRA) along with chemotherapy is superior to ATRA alone. Am. J. Hematol. 1999, 60, 87-93. [CrossRef]

95. Hao, L.; Zhao, J.; Wang, X.; Wang, H.; Wang, H.; Xu, G. Hepatotoxicity From Arsenic Trioxide for Pediatric Acute Promyelocytic Leukemia. J. Pediatr. Hematol. 2013, 35, 67-70. [CrossRef] [PubMed]

96. Teva Announces U.S. FDA Approval of TRISENOX®(arsenic trioxide) Injection for First Line Treatment of Acute Promyelocytic Leukemia. Available online: https://ir.tevapharm.com/news-and-events/press-releases/ press-release-details/2018/Teva-Announces-US-FDA-Approval-of-TRISENOX-arsenic-trioxide-Injectionfor-First-Line-Treatment-of-Acute-Promyelocytic-Leukemia/default.aspx (accessed on 15 October 2020).

97. Dinardo, C.D.; Ky, B.; Vogl, D.T.; Forfia, P.; Loren, A.; Luger, S.; Mato, A.; Tsai, D.E. Differentiation syndrome in non-M3 acute myeloid leukemia treated with the retinoid X receptor agonist bexarotene. Med Oncol. 2008, 25, 299-302. [CrossRef]

98. Gniadecki, R.; Assaf, C.; Bagot, M.; Dummer, R.; Duvic, M.; Knobler, R.; Ranki, A.; Schwandt, P.; Whittaker, S. The optimal use of bexarotene in cutaneous T-cell lymphoma. Br. J. Dermatol. 2007, 157, 433-440. [CrossRef]

99. NCI Drug Dictionary-Realgar-Indigo Naturalis Formulation. Available online: https://www. cancer.gov/publications/dictionaries/cancer-drug/def/realgar-indigo-naturalis-formulation (accessed on 15 October 2020).

100. Fukasawa, H.; Nakagomi, M.; Yamagata, N.; Katsuki, H.; Kawahara, K.; Kitaoka, K.; Miki, T.; Shudo, K. Tamibarotene: A Candidate Retinoid Drug for Alzheimer's Disease. Biol. Pharm. Bull. 2012, 35, 1206-1212. [CrossRef]

101. A Biomarker-Directed Phase 2 Trial of SY-1425 in Patients With Acute Myeloid Leukemia or Myelodysplastic Syndrome. Available online: https://clinicaltrials.gov/ct2/show/NCT02807558 (accessed on 15 October 2020).

102. Sanford, D.; Lo-Coco, F.; Sanz, M.A.; Di Bona, E.; E Coutre, S.; Altman, J.K.; Wetzler, M.; Allen, S.L.; Ravandi, F.; Kantarjian, H.; et al. Tamibarotene in patients with acute promyelocytic leukaemia relapsing after treatment with all-transretinoic acid and arsenic trioxide. Br. J. Haematol. 2015, 171, 471-477. [CrossRef]

103. Available online: https://www.ncbi.nlm.nih.gov/pubmed. (accessed on 10 November 2019).

104. Stahl, M.; Tallman, M.S. Differentiation syndrome in acute promyelocytic leukaemia. Br. J. Haematol. 2019, 187, 157-162. [CrossRef] [PubMed]

Publisher's Note: MDPI stays neutral with regard to jurisdictional claims in published maps and institutional affiliations.

(C) 2020 by the authors. Licensee MDPI, Basel, Switzerland. This article is an open access article distributed under the terms and conditions of the Creative Commons Attribution (CC BY) license (http://creativecommons.org/licenses/by/4.0/). 